\title{
Solenoidal ionization cooling lattices
}

\author{
R. C. Fernow and R. B. Palmer \\ Brookhaven National Laboratory, Upton, New York 11973, USA
}

(Received 21 February 2007; published 12 June 2007)

\begin{abstract}
We explore a practical approach for designing ionization cooling channels with periodic solenoidal focusing. We examine the lattice characteristics in terms of the properties of the coils and the cell geometry. The peak magnetic field in the coils is an important engineering constraint in lattice design. We examine the dependence of the peak field, momentum passband locations, and the beta function on the coil parameters. We make a systematic examination of all allowed lattice configurations taking into account the symmetry properties of the current densities and the beta function. We introduce a unique classification for comparing cooling lattice configurations. While solutions with a single coil per cell illustrate most of the effects that are important for cooling channel design, the introduction of additional coils allows more flexibility in selecting the lattice properties. We look at example solutions for the problem of the initial transverse cooling stage of a neutrino factory or muon collider and compare our results with the properties of some published cooling lattice designs. Scaling laws are used to compare solutions from different symmetry classes.
\end{abstract}

DOI: 10.1103/PhysRevSTAB.10.064001

PACS numbers: 29.27.- a, 41.85.-p

\section{INTRODUCTION}

Ionization cooling [1,2] is an essential feature of most designs for neutrino factories [3] and muon colliders [4]. The phase space of the muon beam that comes from pion decays greatly exceeds the acceptance of downstream accelerator systems, so a cooling channel is usually included to reduce the transverse emittance. For the initial cooling of the muon beam, the only cooling process that is fast enough compared to the muon lifetime is ionization cooling. In the process of ionization cooling muons are focused onto an absorber in the beam path. The particles lose both transverse and longitudinal momentum while crossing the absorber. The beam is then passed through an rf cavity that only restores the lost longitudinal momentum. The net result is that a particle loses some of its transverse momentum and transverse cooling takes place. The cooling effect is balanced against multiple scattering, which acts to increase the emittance. The relative effect of multiple scattering is reduced if the beam is strongly focused in the absorber. Thus, we see that a transverse cooling channel has three essential ingredients: an absorber, an rf cavity, and a system of some sort to focus the beam in the absorber and confine it during its transport through the cavity.

A periodic lattice of solenoid magnets is often used for focusing in linear cooling channels. One has the freedom here to place coils in optimal locations to achieve specific design goals. The use of smaller radius solenoids in specific locations results in less expensive designs than a continuous solenoid channel. In addition, if the polarity of the solenoids alternate along the lattice, there is no buildup of canonical angular momentum. The chief disadvantage of the periodic arrays is the presence of

*fernow@bnl.gov momentum stop bands, which restrict the usable operating range.

A number of cooling lattices using periodic solenoid focusing have been designed over the past decade. Historically the first system considered had a simple sinusoidal dependence of the longitudinal field with axial position. In analogy with the common FODO channel of quadrupole lenses, this was designated a focusing-driftfocusing-drift (FOFO) lattice [5] since each solenoid lens focuses both transverse planes simultaneously. Later, following a suggestion by Andrew Sessler, it was found that the performance of these lattices could be improved significantly by the addition of higher harmonic terms to the on-axis fields [6]. Lattices of this type were given the name "super-FOFO" or "SFOFO." By changing the coil configurations and symmetry properties a number of periodic lattice configurations were discovered. These were typically given their own unique names. One problem with this development was that the relation of these solutions to each other was not always clear. Another problem was that the chosen names were often confusing and not uniquely defined.

Some of the basic properties of a periodic solenoid lattice can be qualitatively determined with a thin lens analysis [7,8]. A step beyond this was made by Penn $[9,10]$, who examined periodic solenoidal lattices in terms of the addition of second and third harmonics to the fundamental sinusoidal field. This analysis introduced a scaling variable $\chi=\frac{B_{0} \lambda}{p}$, where $B_{0}$ is the peak on-axis field, $\lambda$ is the period of the magnetic field, and $p$ is the momentum of the particle. Stable lattice configurations could be located on phase diagrams with $\chi$ as the abscissa and the relative amount of harmonic content as the ordinate. This type of analysis was later extended and the stable regions of the space were more clearly identified [11]. A 
limitation with this approach is that the magnetic fields used in most of the cooling lattices adopted in practice, such as the lattice for the U.S. Study 2 neutrino factory, have much more complex Fourier decompositions.

An alternative analysis of lattice stability and beta function symmetries has been done using recursive solutions of Hill's equation [12]. This method uses Fourier coefficients of the focusing function as input. It produces power series expansions for the trace of the one-cell transport matrix as a function of momentum and for the beta function as a function of position. It was found to produce results in good agreement with the simpler method that is used in this paper.

While an analysis of lattice properties in terms of its leading harmonic content gives some important theoretical understanding of its characteristics, it is not the most practical approach for designing a cooling channel. In this paper we look instead at lattice characteristics in terms of the properties of the coils and the cell geometry. We use the number of coils in a geometric cell and the symmetry properties of the current densities and the beta function to introduce a unique classification for comparing cooling lattice configurations. We separate the results presented here by the number of coils per geometric cell. We first examine solutions with a single coil per cell. This arrangement illustrates most of the effects that are important for cooling channel design. Then we examine the changes that are introduced as additional coils are added to the cell.

\section{IONIZATION COOLING LATTICES}

The only practical method for initial cooling of muon beams is ionization cooling. Typical applications such as neutrino factories [3] require the cooled muon beam to have $\epsilon_{T N} \sim 7 \mathrm{~mm} \mathrm{rad}$, while muon colliders [4] need $\epsilon_{T N} \sim 0.05 \mathrm{~mm} \mathrm{rad}$.

\section{A. Properties of cooling lattices}

In ionization cooling the fractional change in emittance is proportional to the fractional change in momentum arising from energy loss. Multiple scattering in the absorber material is a competing process that acts to increase the transverse emittance. The balance between the strength of these two processes determines whether net cooling takes place.

When the competing processes of energy loss and multiple scattering become equal, the beam reaches an equilibrium normalized transverse emittance given by

$$
\epsilon_{T N}^{\mathrm{eq}} \approx \frac{\beta_{T} E_{S}^{2}}{2 \beta m c^{2} L_{R}\left|\frac{d E}{d x}\right|}=\frac{\beta_{T}}{\beta} C,
$$

where $\beta_{T}$ is the beta function from the focusing system, $E_{S}=14.1 \mathrm{MeV}, \beta$ is the relativistic velocity factor, $m c^{2}$ is the muon rest mass, and $L_{R}$ and $d E / d x$ are the radiation length and ionization energy loss rate of the absorber material. We have collected the material-dependent properties into the quantity $C$. One wants to use absorbers where the product of radiation length and the energy loss rate is large. Hydrogen and lithium hydride are the best choices. The only other parameter that is under our control is the beta function, which we want to keep as small as possible over the length of the absorber.

Transverse ionization cooling can take place in principle at any momentum. However, at low momentum the slope of the $\frac{d E}{d x}$ curve causes lower momentum particles to lose more energy than higher energy particles. This increases the energy spread and leads to a blowup in the longitudinal emittance. Cooling at high momentum is uneconomical since a lot of rf power is required to replace a fixed fraction of the initial energy. For these reasons cooling channels are typically designed with a reference momentum near the minimum of the $\frac{d E}{d x}$ curve $(\approx 200 \mathrm{MeV} / c)$. The momentum is sometimes reduced in the final stages of the channel in order to reduce the value of the beta function and to take advantage of the higher value of $\frac{d E}{d x}$.

At $200 \mathrm{MeV} / c$, the energy loss rate in liquid hydrogen is $\sim 0.3 \mathrm{MeV} / \mathrm{cm}$. If the beta function can be kept small over $\sim 30 \mathrm{~cm}$, the total loss of energy is $9 \mathrm{MeV}$. For a $200 \mathrm{MHz}$ rf cavity with a gradient of $15 \mathrm{MV} / \mathrm{m}$ and operating $30^{\circ}$ off the zero crossing, we would need $\sim 1.2 \mathrm{~m}$ of cavities to replace the lost energy. Thus most of the space in a typical cell in a cooling lattice is taken up with rf cavities.

A solenoidal focusing system in a cooling channel has a large number of sometimes conflicting requirements. For example, the minimum value of the beta function should be small over an axial region longer than the absorber, the maximum value of the beta function should be small for reasonable transverse beam apertures, and the momentum acceptance of the lattice should overlap the reference momentum and be larger than the momentum spread of the desired beam $(\sim \pm 30 \%$ full width). For efficient cooling the channel must have very large angular acceptance. Field reversals are necessary to prevent the buildup of canonical angular momentum. Energy straggling and transverse-longitudinal coupling should not cause loss of particles from the rf bucket.

There are also engineering constraints on any practical magnetic configuration. The operating current in a superconducting magnet must be smaller than the critical current corresponding to the peak field in the coil, the arrangement of coils must allow access to the rf cavities and absorbers, and there may be constraints on the strength and direction of the magnetic field in the rf cavities to prevent breakdown from limiting the useful electric field gradient in the cavity.

Cooling channels differ from most other beam transport systems because they must transmit large divergence, i.e., nonparaxial, particles. In order for the rate of beam heating to be small, the mean beam divergence should be much larger than the mean multiple scattering angle. For efficient 
cooling the channel needs to transmit rms angles at least twice as large as the equilibrium value. If the full acceptance required is 3 times the rms value, we find the required angular acceptance is [13]

$$
A_{\theta} \approx 3 \sqrt{\frac{2 C}{\beta^{2} \gamma}} .
$$

For $200 \mathrm{MeV} / c(\beta=0.89, \gamma=2.14)$ and liquid hydrogen $\left(C=4.2 \times 10^{-3}\right)$, we find the required angular acceptance is $\sim 210 \mathrm{mrad}$.

Angular momentum is a complication of solenoid channels that is not encountered in other focusing systems [14]. This effect can be eliminated by placing absorbers at locations where $B_{z}=0$ or by alternating the direction of the magnetic field. This can be done most naturally by using alternating-polarity lattices where the field reverses in alternate cells.

Solenoid lattice design has traditionally been done by varying the parameters of a few coils at the boundary between lattice cells, such that beta function is preserved over a range of incident momentum values. In practice, a computer program was used to minimize the beta function slopes at the cell boundary at, for example, 9 momenta spaced at $10 \%$ intervals around the central value. Although this method has been very successful in producing useful lattice designs, there is always the danger that an even better design may have been overlooked. In addition, many solenoid lattice designs found this way have had unacceptably large values of the peak field in the coils. For that reason we explore a more systematic approach to solenoid lattice design in this paper. The new optimization technique places primary emphasis on obtaining a momentum passband for the lattice at a desired reference momentum. Secondary fitting criteria include minimizing the peak field in the coil, maximizing the momentum acceptance, and minimizing the beta function at the reference momentum. Results from this analysis are presented in Secs. V, VI, and VII.

\section{B. Classification of periodic solenoid lattices}

One of our goals is to develop a systematic method for comparing the properties of various periodic solenoid lattices. We define the geometric cell length to be $d$. We assume the focusing is done with one or more solenoids in each cell. We allow the possibility that the current in these solenoids can have different polarities. We also allow that the overall polarity of the magnetic field may alternate between adjacent geometric cells. If $\lambda$ is the period of the magnetic field, then we either have the case $\lambda=d$ when the magnetic field is the same in every geometric cell, or $\lambda=2 d$ when the polarity alternates. We will give prescriptions below for defining the boundary $(b)$ locations between geometric cells, depending on the number of coils per cell. We define the midpoint $(m)$ of a cell to be the axial position midway between the boundaries. We will see that it is useful to maintain this distinction since the lattice solutions with beta minimums at $b$ and $m$ can have very different behavior.

The beta function depends on the geometry of the lattice, the symmetry of the magnetic field, and on the reference momentum of the particles of interest. For cooling lattices we are mainly interested in the spatial location and size of the minimum of the beta function, since these points determine where the absorbers should be located. We will see that periodic solenoid lattices have a series of momentum passbands. We will denote the high momentum passband as number 1 and then count the other passbands in order as we decrease the momentum. The spatial location of the minimum of the beta function changes for each passband. We are primarily interested in solutions with the minimum located either at the cell boundary or at the midpoint of the cell. Solutions with multiple minima in the cell interior are also possible, but these do not appear to offer any particular advantages for cooling purposes.

In order to compare the properties of various lattice designs, we need a labeling system that uniquely incorporates this lattice information. We denote cooling lattices with symbols that show the relative polarity of the coils in adjacent geometric cells, the location of the minimum of the beta function, and the number of the momentum passband for the reference momentum. We choose symbols that have a beta minimum in the center so that the coil arrangement on either side of the minimum is immediately apparent.

For solutions with the minimum of the beta function at the cell boundary we arbitrarily choose the first coil to have positive polarity. The boundary between the two geometric cells is denoted with a vertical line and we append the number of the passband as a subscript at the end of the symbol. Thus, for example, a lattice with 1 coil per geometric cell, alternating polarity in adjacent geometric cells,
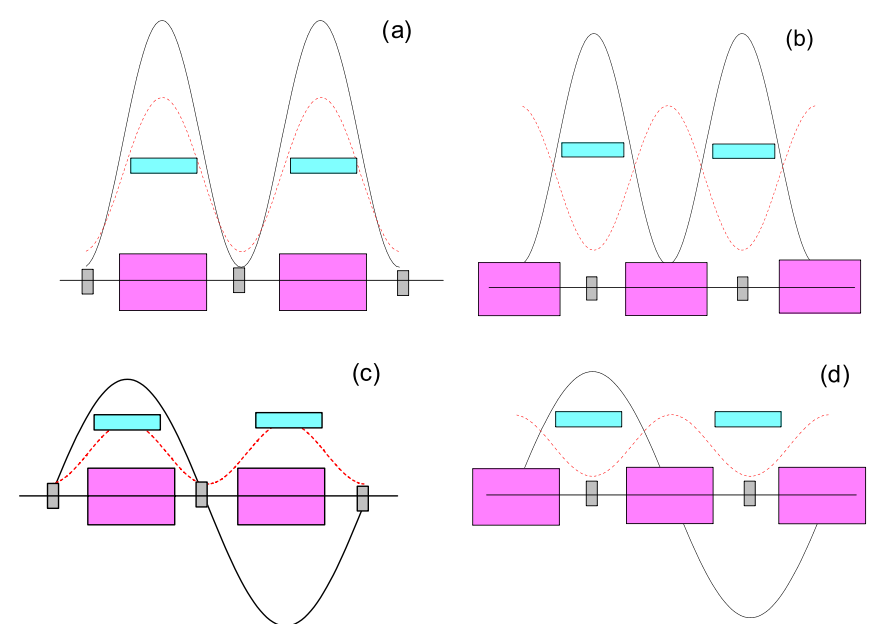

FIG. 1. (Color) The four basic lattice configurations for one coil per cell. (a) $\langle+\mid+\rangle$, (b) $\langle+\hat{+}+\rangle$, (c) $\langle+\mid-\rangle$, (d) $\langle-\hat{+}-\rangle$. 
with the beta minimum at the cell boundary, and operation in the high-energy passband would be denoted $\langle+\mid-\rangle_{1}$.

For solutions with the minimum of the beta function at the cell midplane, the symbol shows the polarity of the coils in a complete cell together with the polarity of the coils up to the center of the two adjacent half-cells. We use a caret symbol to indicate the location of the minimum of the beta function. For example, for a lattice with 3 coils of the same polarity per geometric cell, alternating polarity in adjacent geometric cells, with the beta minimum at the cell midplane, and operation in the second passband, the symbol would be $\langle--+\hat{+}+--\rangle_{2}$. For lattices with an odd number of coils per cell the minimum of the beta function would be located under one of the coils.

Figure 1 shows the four basic configurations for the case when there is one coil per cell. In this figure the rf cavities are colored magenta, the absorbers are gray, and the solenoid coils are cyan. The black solid curve is the magnetic field and the red dotted curve is the beta function.

\section{SOLENOID PROPERTIES}

We gather here several properties of solenoid magnets that we will require later in the paper. The focal length $f$ of a thin solenoid lens is given by [15]

$$
f=\frac{4 p^{2}}{e^{2} B_{0}^{2} L},
$$

where $p$ is the particle momentum, $B_{0}$ is the solenoid field strength, and $L$ is the length of the solenoid. The beta function of a uniform solenoid channel has the value

$$
\beta_{o}=\frac{2 p}{e B}
$$

inside the solenoid. We note that the beta function can be reduced by decreasing the operating momentum or by increasing the solenoid field strength.

For the computer calculations, we use a cylindrical block description of a solenoidal coil. The coil has a total length $L$, inner radius $a$, outer radius $b$, and carries the current density $J$. If $z$ is the axial position of an observation point measured from the center of the solenoid, then the on-axis field is given by

$$
\begin{aligned}
B(z)= & \frac{\mu_{0} J}{2}\left\{(L-z) \ln \left[\frac{b+\sqrt{b^{2}+(L-z)^{2}}}{a+\sqrt{a^{2}+(L-z)^{2}}}\right]\right. \\
& \left.+(L+z) \ln \left[\frac{b+\sqrt{b^{2}+(L+z)^{2}}}{a+\sqrt{a^{2}+(L+z)^{2}}}\right]\right\} .
\end{aligned}
$$

This description of the solenoidal field will be used later in determining the beta functions of the lattices.

In order to investigate the peak fields in the coils, we need off-axis expressions for the solenoid fields. In general, both longitudinal and radial field components are present. The field component for current sheets can be expressed in closed form in terms of elliptic integrals [16]. Let us define the functions

$$
\begin{aligned}
& b_{z}(r, z)=\frac{\mu_{0} I^{\prime}}{\pi} \frac{z a}{\zeta(a+r)}\left[K(k)+\frac{a-r}{2 a}(\Pi(k, c)-K(k))\right] \\
& b_{r}(r, z)=\frac{\mu_{0} I^{\prime}}{\pi} \frac{\zeta}{4 r}\left[2(K(k)-E(k))-k^{2} K(k)\right],
\end{aligned}
$$

where $I^{\prime}$ is the current per unit length and we use the auxiliary quantities

$$
\begin{gathered}
k=\sqrt{\frac{4 a r}{(a+r)^{2}+z^{2}}} \quad \zeta=\sqrt{(a+r)^{2}+z^{2}} \\
c=-\frac{4 a r}{(a+r)^{2}} .
\end{gathered}
$$

$K(k), E(k)$, and $\Pi(k, c)$ are complete elliptic integrals. The magnetic field from the solenoidal sheet is given in terms of these functions by

$$
\begin{aligned}
& B_{z}(r, z)=-b_{z}(r, z-L)+b_{z}(r, z+L) \\
& B_{r}(r, z)=b_{r}(r, z-L)-b_{r}(r, z+L) .
\end{aligned}
$$

We approximate the field of the current block by using the sum of the fields from 10 current sheets with different radii.

\section{PERIODIC SOLENOID LATTICES}

For the remainder of this paper we will consider lattices with periodically varying solenoidal fields. If we consider a particle whose energy is high enough, the period of the particle's betatron oscillations $\Lambda$ is longer than the geometric cell length of the lattice $d$. As the momentum of the particle is reduced, we will eventually reach the point when $\Lambda=d$. At this point the particle sees the same radial kick at the corresponding locations in each oscillation and a resonance causes the amplitude of the motion to grow. Under these conditions the particle has a phase advance of $\pi$, so we call this the $\pi$ resonance. If the momentum continues to be reduced, we will reach the condition that the particle makes two betatron oscillations while traversing each cell of the lattice and a second $(2 \pi)$ resonance occurs. In general resonances occur whenever $\lambda=n d$, where $n$ is an integer.

\section{A. Mathieu analysis}

Stable solutions for the motion of charged particles in lattices with sinusoidally varying magnetic fields can be found from the eigenvalues of the Mathieu equation [1720]. The radial and azimuthal equations of motion of a charged particle are given in cylindrical coordinates as

$$
\ddot{r}-r \dot{\phi}^{2}=\frac{e r \dot{\phi} B_{z}}{m \gamma} \quad \frac{m \gamma}{r}\left(r^{2} \ddot{\phi}+2 r \dot{r} \dot{\phi}\right)=-q \dot{r} B_{z},
$$


TABLE I. Mathieu stop bands.

\begin{tabular}{lrr}
\hline \hline Stop band & $q$ (low) & $q$ (high) \\
\hline 1 & 0.3290 & 0.8898 \\
2 & 1.8582 & 3.0391 \\
3 & 4.6270 & 6.4259 \\
4 & 8.6316 & 11.0480 \\
5 & 13.8711 & 16.9047 \\
\hline \hline
\end{tabular}

where the dots are time derivatives and we neglect any changes in $\gamma$. If we assume that the azimuthal acceleration can be neglected, the azimuthal equation reduces to

$$
\dot{\phi}=-\frac{q B_{z}}{2 m \gamma} .
$$

Requiring this constraint decouples the radial and azimuthal motions. This is known as the Larmor frame of reference. In a constant solenoid field in the laboratory frame the Larmor frame rotates with a constant angular velocity. In the case here with a periodically reversing field the Larmor frame oscillates back and forth. The radial equation becomes

$$
\ddot{r}+\frac{r}{4}\left(\frac{e B_{z}}{m \gamma}\right)^{2}=0 .
$$

If we now assume that near the axis

$$
B_{z}(z)=B_{0} \sin (k z)
$$

we find the radial equation

$$
\ddot{r}+r\left(\frac{e B_{0}}{2 m \gamma}\right)^{2} \sin ^{2}(k z)=0 .
$$

This equation can be transformed into the canonical form of the Mathieu equation

$$
\frac{d^{2} y}{d \nu^{2}}+[a-2 q \cos (2 \nu)] y=0
$$

The parameter

$$
q=\left(\frac{e B_{0} \lambda}{8 \pi p}\right)^{2}
$$

where $\lambda$ is the period of the magnetic field and $p$ is the momentum of the particle. For the case considered here the parameter $a=2 q$. Stable, periodic solutions of the Mathieu equation only exist for certain values of the parameter $q$.

The first five stop bands for particle momentum in a sinusoidally varying solenoid field are given in Table I. We will use these results in the following section to check the accuracy of our computer calculations of the lattice properties.

\section{B. Symplectic integrator method}

We can examine the properties of finite size, i.e., nonthin lens, coils by evaluating the one-cell transport matrix numerically using a second order symplectic integration algorithm [21]. We break the cell up into a large number of parts, each part consisting of a half-step of drift, a thin lens and another half-step of drift. The focusing strength $K$ of the thin lens is determined from the local value of the onaxis solenoid field from

$$
K(z)=\left(\frac{e B_{z}(z)}{2 p}\right)^{2} .
$$

The accuracy of this method is very good as we demonstrate below in comparisons of the tracking results with the predictions of the Mathieu theory for lattices with sinusoidal magnetic fields.

Note that the focusing strength is proportional to $B^{2}$. Unlike quadrupoles, solenoids focus the beam regardless of the direction of the field. There are, however, differences in angular beam dynamics since reversing the field causes the particles to rotate in opposite directions, or equivalently it reverses the direction of the angular momentum. These differences affect the radial dynamics only if transverse fields are introduced, e.g., to obtain dispersion.

\section{Peak field}

The range of allowed coil parameters is strongly constrained by the field enhancement on the solenoid coil. The peak field on the coil usually occurs near the inner surface. In the design work here, we try to keep the engineering current density at or below $100 \mathrm{~A} / \mathrm{mm}^{2}$ at $4.2 \mathrm{~K}$. This limits the peak field to $\sim 8 \mathrm{~T}$ for NbTi coils and $\sim 17 \mathrm{~T}$ for $\mathrm{Nb}_{3} \mathrm{Sn}$ coils [22]. In addition there are stress limitations that constrain the coil parameters [23]. A rule of thumb in solenoid design is that the criterion

$$
\sigma_{p}[\mathrm{MPa}]=B_{z}[\mathrm{~T}] r[\mathrm{~m}] J\left[\frac{\mathrm{A}}{\mathrm{mm}^{2}}\right]<350
$$

should be satisfied for all locations in the coil, where $\sigma_{p}$ is the peak stress, $B_{z}$ is the field in the coil at the radius $r$, and $J$ is the current density. If this criterion is violated, it is likely that the conductor will not be able to support the magnetic stresses without the addition of high-strength nonconducting support layers.

Purely sinusoidal field lattices have a severe problem with peak fields in large-radius solenoid coils [24]. An exact solution of the field using Maxwell's equations in cylindrical coordinates can be written as

$$
\begin{aligned}
& B_{z}(r, z)=A \cos \left(\frac{2 \pi z}{\lambda}\right) I_{0}\left(\frac{2 \pi r}{\lambda}\right) \\
& B_{r}(r, z)=A \sin \left(\frac{2 \pi z}{\lambda}\right) I_{1}\left(\frac{2 \pi r}{\lambda}\right),
\end{aligned}
$$

where $I_{0}$ and $I_{1}$ are modified Bessel functions. For ex- 


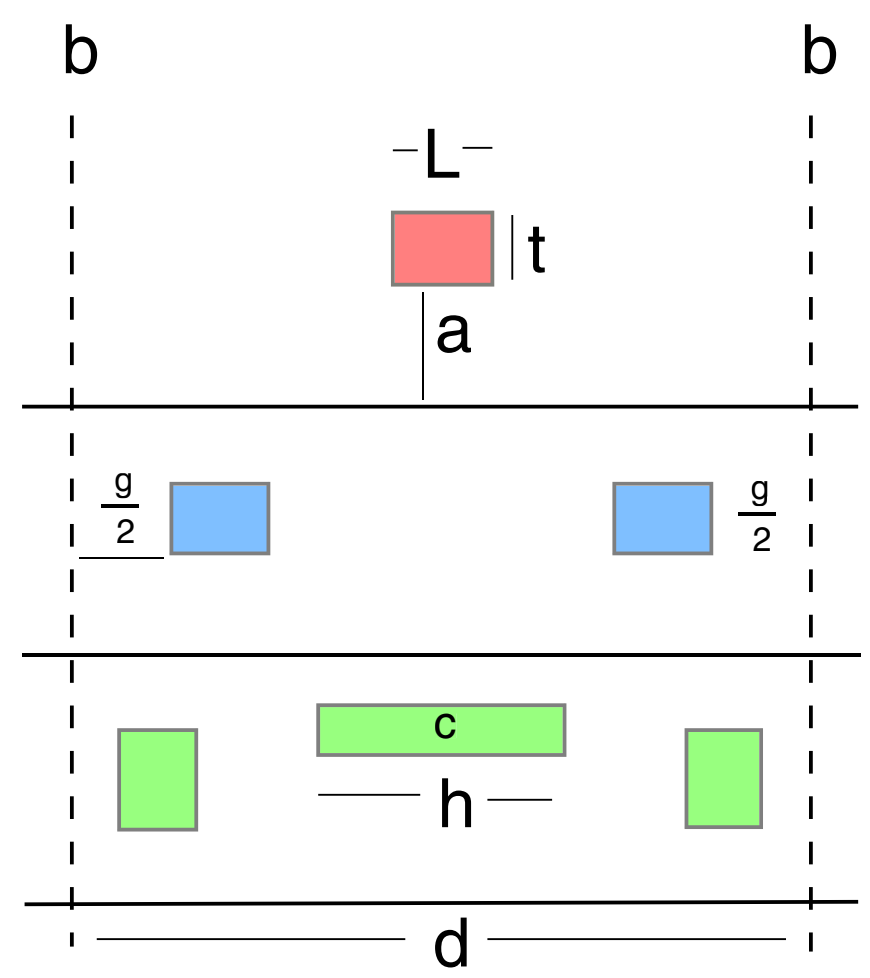

FIG. 2. (Color) Basic configuration of lattices with one, two, and three coils per cell.

ample, at a radius equal to the cell length (half the period of the magnetic field $\lambda$ ), the value of the function $I_{0}$ is 5.5 , the value of the function $I_{1}$ is 4.5 , and the peak field is $\sim 7$ times the desired field value $A$ on the axis.

\section{Representative lattices}

The lattice configurations considered in this paper are illustrated in Fig. 2. The cell boundaries are marked $b$. For one coil per cell, we use $L$ for the coil length, $a$ for the inner radius, and $t$ for the radial thickness. This also applies to the two symmetric coils nearest the boundaries in the two and three coil per cell cases. The distance across the boundary to the corresponding coil in the next cell is $g$. For the three coil per cell lattice the additional coil is denoted $c$. It is centered between the boundaries and can have different dimensions than the other coils. The length of the $c$ coil is denoted $h$.

In order to examine specific examples of the various classes of possible lattices, we need to select a particular cooling objective. In the following we look for solutions that would be suitable for the early stages of a neutrino factory or muon collider. A typical reference momentum is $200 \mathrm{MeV} / c$. We will try to provide as large a momentum acceptance as possible. The beam radius is typically $30 \mathrm{~cm}$, so we set a lower limit on the inner radius of the coil of $35 \mathrm{~cm}$. Examples of this type of channel use rf cavities with frequency around $200 \mathrm{MHz}$, which has a radius $\sim 60 \mathrm{~cm}$. We take the length of the cavity to be at least $50 \mathrm{~cm}$. We can satisfy these conditions either by using short coils of small radius located between cavities, or by using longer coils with large radius located around the rf cavity.

\section{LATTICES WITH ONE SOLENOID PER CELL}

We now consider cooling lattices with one solenoid coil in each geometric cell. The four characteristic designs of this type were illustrated in Fig. 1. There are five continuous parameters and one symmetry factor $\left\{d, L, a, t, J ; f_{e}\right\}$ that determine the properties of these lattices. We will use the external symmetry parameter $\left(f_{e}\right)$ to indicate whether the polarity of the current in the coils alternate in neighboring geometric cells. We use $f_{e}=-1$ if the polarity flips and $f_{e}=+1$ if the polarity remains the same. Solutions must obviously satisfy the constraint $L<d$. We define the cell boundary $(b)$ to be the axial location midway between the coils and the cell midplane $(\mathrm{m})$ to be the axial position through the center of the coil.

\section{A. Thin lens approximation}

Let us start by examining the lattice using the thin lens approximation. This analysis is applicable to the solutions with minimums of the beta function at the cell boundary. Consider the case where we begin the matrix calculation in the center of one of the solenoids. Then the one-cell transport matrix $M$ is the product

$$
M=F\left(\frac{f}{2}\right) D(d) F\left(\frac{f}{2}\right),
$$

where $F$ is the $2 \times 2$ matrix for a thin lens of focal length $f / 2$ and $D$ is the matrix for a drift space of length $d$. This matrix is then compared to the standard form of the Courant-Snyder matrix

$$
\left|\begin{array}{cc}
\cos \psi+\alpha \sin \psi & \beta \sin \psi \\
-\gamma \sin \psi & \cos \psi-\alpha \sin \psi
\end{array}\right| .
$$

This allows us to immediately determine the relations for the phase advance per cell and the maximum value of the beta function

$$
\cos \psi=1-\frac{d}{2 f} \quad \beta_{\max }=\frac{d}{\sin \psi} .
$$

The minimum value of $\beta_{\max }$ occurs when the phase advance is $\pi / 2$. The scale of the magnitude of the beta function is set by the cell length $d$. We can find an expression for the minimum value of the beta function by calculating a new matrix beginning midway between the solenoids

$$
M=D\left(\frac{d}{2}\right) F(f) D\left(\frac{d}{2}\right)
$$

This gives 


$$
\beta_{\min }=\frac{d}{2 \sin \psi}(1+\cos \psi) .
$$

If we now use the expression for the focal length of the thin solenoid lens from Eq. (2), we find that the phase advance per cell is given by

$$
\cos \psi=1-\frac{e^{2} B_{0}^{2} L d}{8 p^{2}}
$$

For very high momentum particles the phase advance is $\sim 0$. As $p$ decreases the phase advance reaches a maximum value of $\pi$ when

$$
p_{\pi}=\frac{e B_{0}}{4} \sqrt{L} \bar{d}
$$

Below this momentum, Eq. (5) for the phase advance becomes unphysical and there is a stop band in the momentum spectrum. The minimum value of the beta function approaches 0 at the edge of the stop band. This type of analysis fails when real solenoids have lengths that are a significant fraction of the cell length $d$.

\section{B. Sinusoidal field approximation}

In order to compare the results of our lattice calculations using the symplectic integrator with the Mathieu theory, we first found the single coil configuration that gave the best approximation to a pure sine field on-axis. The results from an optimization program produced a very pure sine wave field. The stability of a solution can be found from the trace of the one-cell transport matrix. Solutions are stable provided the absolute value of the trace is less than 2 . Figure 3 shows a plot of the trace versus momentum for

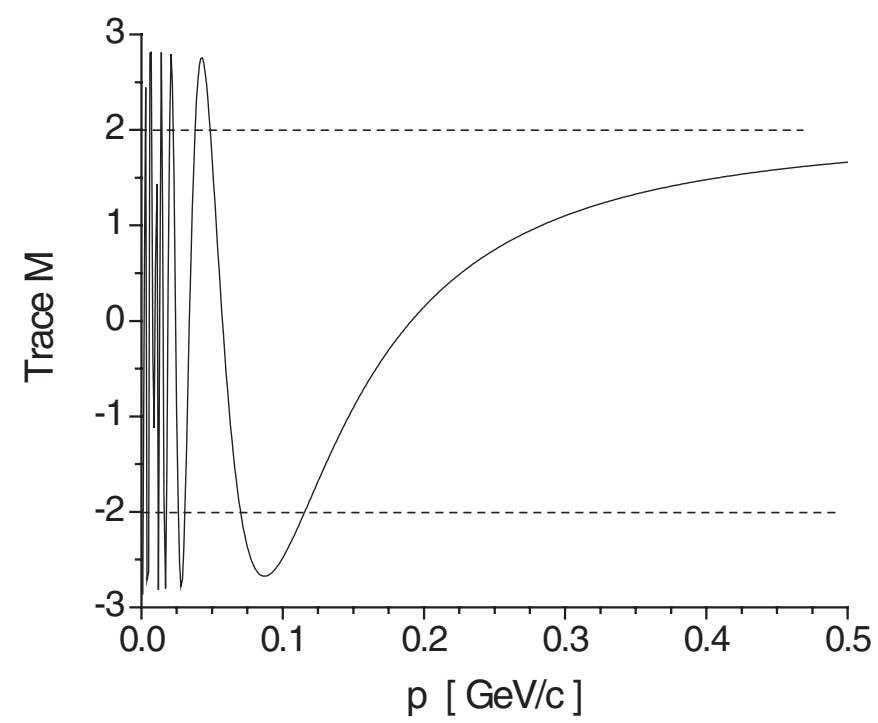

FIG. 3. Trace of the one-cell transport matrix versus momentum. The parameter values were $d=100 \mathrm{~cm}, L=67.1 \mathrm{~cm}$, $a=43.6 \mathrm{~cm}, t=12.6 \mathrm{~cm}, J=40 \mathrm{~A} / \mathrm{mm}^{2}$, and alternating polarity.
TABLE II. Momentum passband locations $[\mathrm{MeV} / c]$.

\begin{tabular}{lcc}
\hline \hline \multicolumn{1}{c}{$q$} & One-coil passband & Mathieu theory \\
\hline $0.329-0$ & $116-\infty$ & $116-\infty$ \\
$1.86-0.890$ & $49-70$ & $49-70$ \\
$4.63-3.04$ & $31-37$ & $31-38$ \\
$8.63-6.42$ & $23-26$ & $23-26$ \\
$13.87-11.05$ & $18-19$ & $18-20$ \\
\hline \hline
\end{tabular}

the sine wave solution. We see that as predicted by the thin lens theory the lattice transmits high momentum particles. As the momentum drops we reach the upper edge of the $\pi$ resonance at $116 \mathrm{MeV} / c$, whereas the thin lens prediction from Eq. (6) is $173 \mathrm{MeV} / c$. However, Fig. 3 also shows additional momentum passbands below $70 \mathrm{MeV} / c$, which were not present in the thin lens theory.

The momentum passband structure from this coil configuration are quantitatively compared with the Mathieu theory results in Table II. The momentum values for Mathieu theory come from the relation

$$
p=\frac{e B_{0} \lambda}{8 \pi \sqrt{q_{M}}}
$$

where $B_{0}=2.78 \mathrm{~T}, \lambda=2 \mathrm{~m}$, and the $q_{M}$ values for the passbands are taken from Table I. The agreement with theory is excellent. The high-energy passband has $\langle+\mid-\rangle_{1}$ symmetry while the second band has $\langle-\hat{+}-\rangle_{2}$ symmetry. Each of the additional lower momentum bands has an additional minimum of the beta function in the interior of the cell.

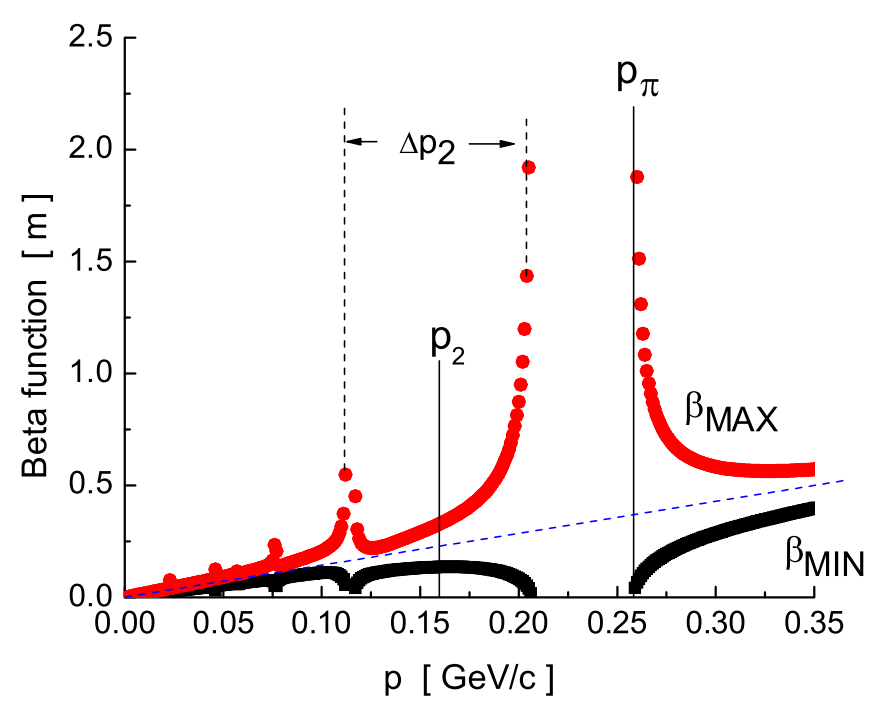

FIG. 4. (Color) Minimum and maximum values of the beta function as a function of $p$. The parameter values were $d=$ $100 \mathrm{~cm}, L=40 \mathrm{~cm}, a=40 \mathrm{~cm}, t=10 \mathrm{~cm}, J=100 \mathrm{~A} / \mathrm{mm}^{2}$, and constant polarity. 


\section{Lattice properties}

The minimum and maximum values of the beta function are shown as a function of $p$ in Fig. 4. At the edge of every stop band, the minimum value of the beta function in the cell approaches 0, while the maximum value of the beta function becomes very large. This general behavior of the beta function as a function of momentum does not depend on the polarity of the magnetic field in alternate cells. In some lattices the axial position where the beta minimum occurs in a given passband changes as the momentum is varied. We define the lower edge of the high-energy passband to be $p_{\pi}$. We are also interested in the possibility of using the second passband for cooling. The beta function is flatter and the maximum value of $\beta_{\min }$ is limited in this passband. We define $p_{2}$ to be the center of this passband and $\Delta p_{2}$ to the full width of the band. The dashed line in the figure shows the linear dependence of the beta function on momentum for a continuous solenoid expected from Eq. (3). Note how the minimum and maximum beta functions in each passband approach the value for the continuous solenoid.

\section{Location of the momentum passbands}

The momentum of the $\pi$ resonance increases with the length of the cell if the coil dimensions remain fixed. The dependence is stronger for the alternating-polarity case. The center of the second passband increases for alternating polarity, but is insensitive to cell length for constant polarity.

Figure 5 shows the momentum dependence as a function of the coil length $L$. The length of the solenoid has a strong effect on the value of $p_{\pi}$, and on the location and width of the second, lower momentum passband for constant polar-

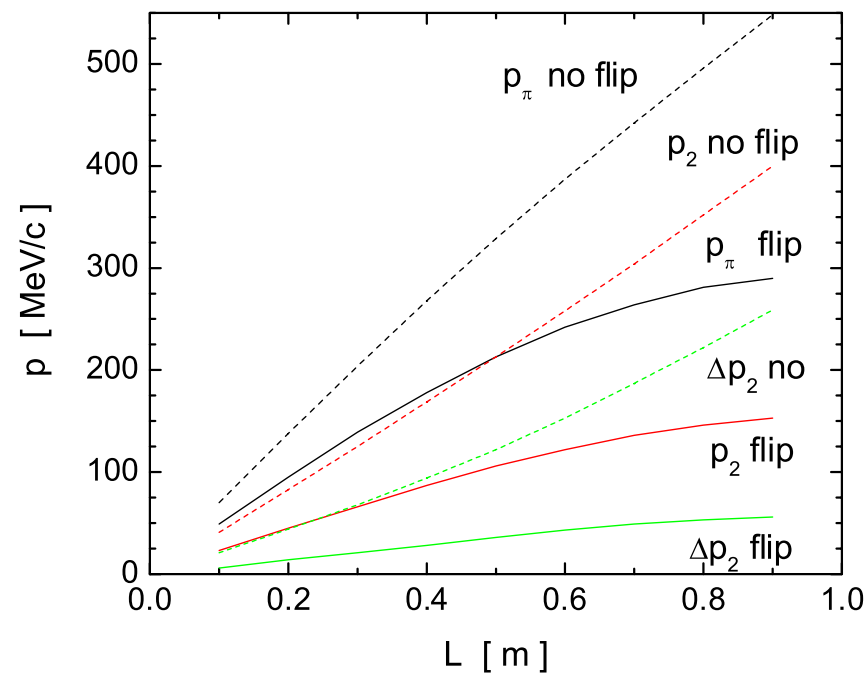

FIG. 5. (Color) Location of the $\pi$ resonance, and the midpoint and width of the second band as a function of $L$. The other parameter values were $d=100 \mathrm{~cm}, a=40 \mathrm{~cm}, t=10 \mathrm{~cm}$, and $J=100 \mathrm{~A} / \mathrm{mm}^{2}$. ity. As $L / d$ increases, the second passband moves up in momentum and expands in acceptance. At $L / d=0.9$ the one-cell transport matrix at the low momentum edge of the second passband just reaches a trace of 2 and the stop bands below this have disappeared. In the limit when $L / d=1$, we have a continuous solenoid and there are no resonances or stop bands. The effect of changing $L$ is somewhat weaker for alternating polarity. The location of $p_{\pi}$ and $p_{2}$ grows at first, but then saturates for the limit $L / d=1$. The width of the second passband $\Delta p_{2}$ never becomes very large.

We next consider changes in the passbands as we vary the radius of the coil. Figure 6 shows the location of the $\pi$ resonance, and the midpoint and width of the second band as a function of the coil radius. Increasing the radius decreases the location of the $\pi$ resonance, especially in the flip case. It also decreases the midpoint of the second band for the flip case. Note, however, that $p_{2}$ is independent of radius for the no-flip case. The width of the second band is largest for large radius coils in the no-flip case.

The location of the $\pi$ resonance, and the midpoint and width of the second band grow linearly with the current density. These quantities also grow approximately linearly with coil thickness.

The location of the $\pi$ resonance can be estimated for alternating-polarity lattices from the Mathieu theory. Since $q_{M}=0.329$ at $p_{\pi}$ and $q_{M} \approx 1.375$ at $p_{2}$, we can use Eq. (7) to find that

$$
p_{\pi}=20.8 B_{0} \lambda \quad p_{2}=10.2 B_{0} \lambda,
$$

where the units are $\{\mathrm{MeV} / c, \mathrm{~T}, \mathrm{~m}\}$. This expression should become more accurate as the field in the lattice approximates a pure sine wave. We have found that $p_{2}$ only scales approximately with $B_{0}$ and that $\Delta p_{2}$ does not scale with $B_{0}$

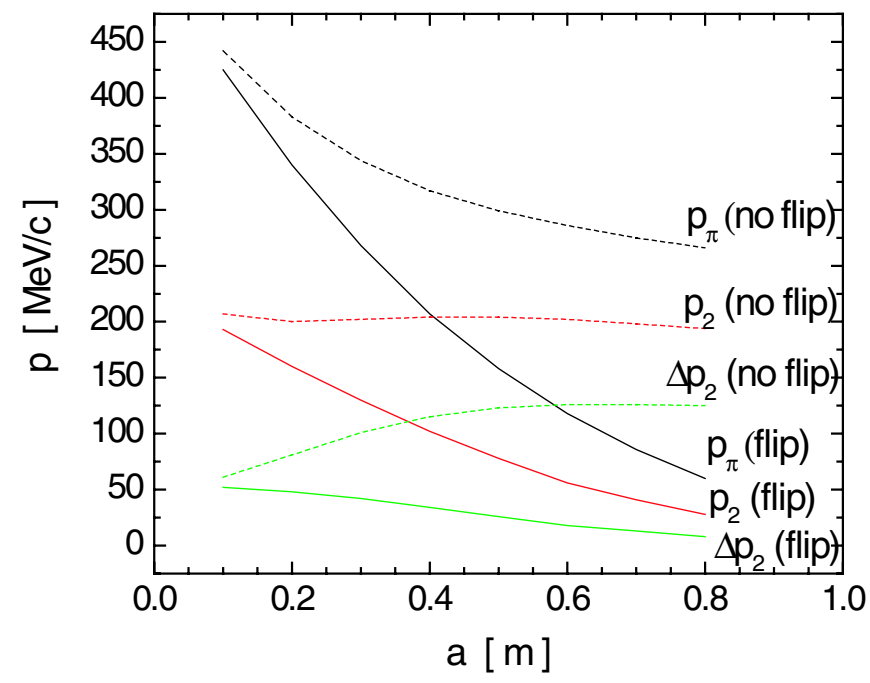

FIG. 6. (Color) Location of the $\pi$ resonance, and the midpoint and width of the second band as a function of the coil radius. The other parameter values were $d=100 \mathrm{~cm}, L=50 \mathrm{~cm}, t=$ $10 \mathrm{~cm}$, and $J=100 \mathrm{~A} / \mathrm{mm}^{2}$. 


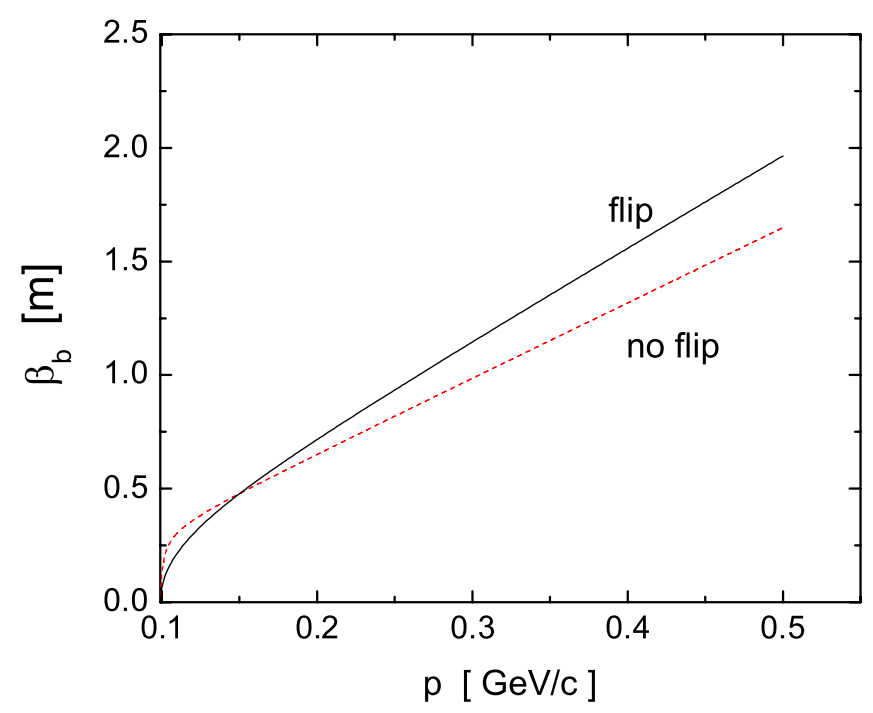

FIG. 7. (Color) Minimum beta function in the high-energy passband versus momentum. The minimum value occurs at the cell boundary.

at all. $B_{0}$ and $p_{\pi}$ have similar dependences on the coil parameters. For very nonsinusoidal fields, e.g., lattices with small $a / d$ and $L / d$ and constant polarity, $p_{\pi}$ is still approximately linearly related to $B_{o}$, but not to $B_{o} d$.

\section{Beta function}

For the high-energy passband, the minimum value of the beta function is determined mainly by the separation of the reference momentum from $p_{\pi}$. This can be seen in Fig. 7 . This figure shows the beta functions for two different coil configurations designed to have $p_{\pi}$ at $100 \mathrm{MeV} / c$. One solution (A1) is a no-flip case, while the other solution (A2) is a flip case. We will discuss the properties of these solutions later in Sec. VE. It is possible to reduce the beta function by moving the reference momentum closer to $p_{\pi}$, but this reduces the momentum acceptance.

We turn now to the minimum value of the beta function evaluated at the center $\left(p_{2}\right)$ of the second passband. We examine the dependence of the solutions on the geometrical parameters under the constraint that $p_{2}$ is fixed. Figure 8 shows the value of the beta function at the cell boundary and cell midplane and the momentum acceptance of the band as we vary the cell length for the noflip case. The other coil dimensions were kept constant. We adjusted the current density in the coils in order to keep $p_{2}$ fixed at $200 \mathrm{MeV} / c$. Note that as the cell length is increased the beta function at the cell boundary increases, but the value of the beta function at the midplane and the momentum acceptance both decrease. The beta function is always smaller at the midplane than at the cell boundary. Similar behavior can be seen for the flip case in Fig. 9. Again we see that as the cell length is increased the beta function at the cell boundary increases, but the value of the beta function at the midplane and the momentum accep-

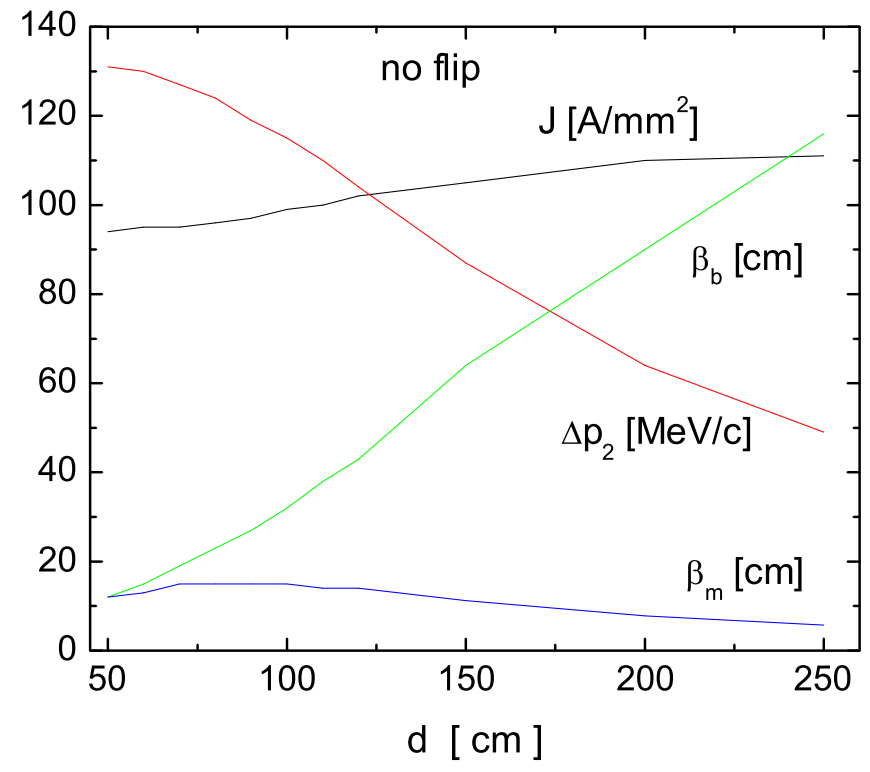

FIG. 8. (Color) Lattice parameters as a function of the cell length for the no-flip case. The beta function at the cell boundary and midplane, momentum acceptance, and current density to keep $p_{2}$ fixed at $200 \mathrm{MeV} / c$ are shown. The other parameter values were $L=20 \mathrm{~cm}, a=40 \mathrm{~cm}$, and $t=24 \mathrm{~cm}$.

tance both fall. The beta function is much smaller at the midplane than at the cell boundary.

There is little dependence of the lattice parameters on the coil length if we require $p_{2}$ to be fixed. The peak field decreases slightly for longer coils. In the limit when $L / d=$ 1 , the value of the beta function for the no-flip case agrees

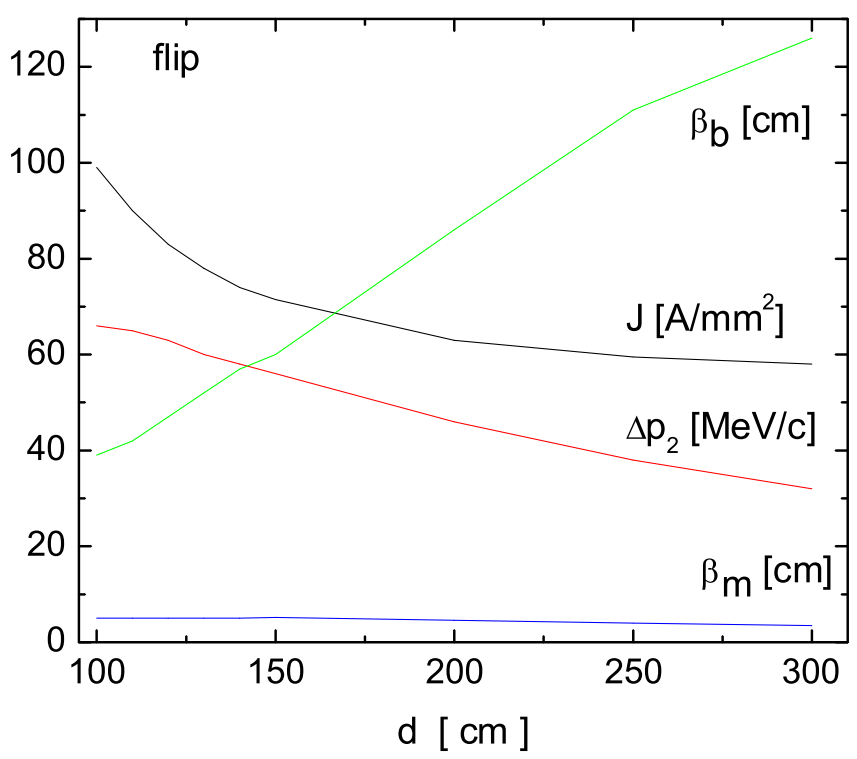

FIG. 9. (Color) Lattice parameters as a function of the cell length for the flip case. The beta function at the cell boundary and midplane, momentum acceptance, and current density to keep $p_{2}$ fixed at $200 \mathrm{MeV} / c$ are shown. The other parameter values were $L=40 \mathrm{~cm}, a=35 \mathrm{~cm}$, and $t=24 \mathrm{~cm}$. 


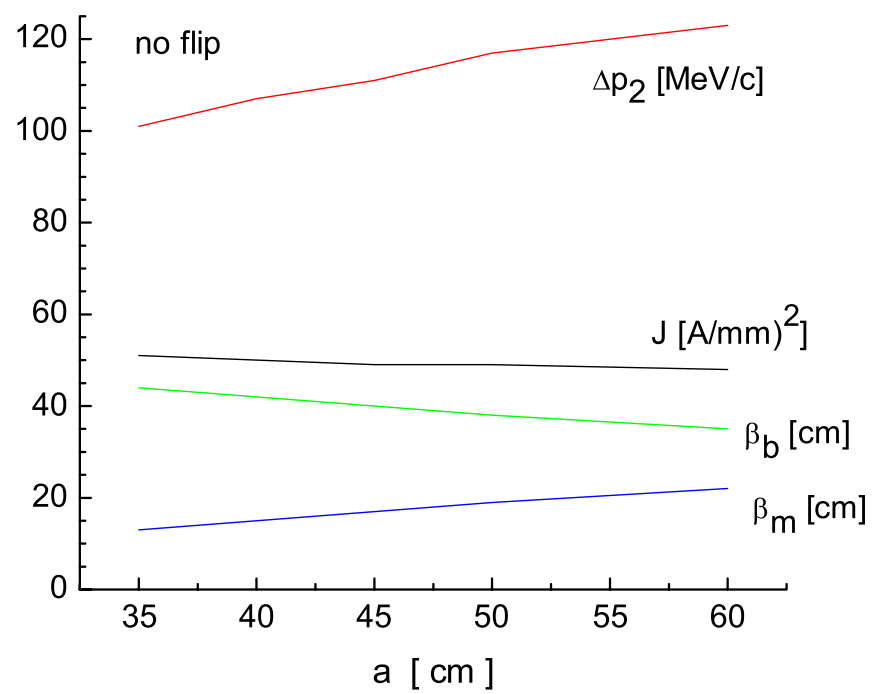

FIG. 10. (Color) Lattice parameters as a function of the solenoid inner radius for the no-flip case. The beta function at the cell boundary and midplane, momentum acceptance, and current density to keep $p_{2}$ fixed at $200 \mathrm{MeV} / c$ are shown. The other parameter values were $d=120 \mathrm{~cm}, L=40 \mathrm{~cm}$, and $t=24 \mathrm{~cm}$.

with Eq. (3) for the continuous solenoid. We show the dependence of the lattice parameters on the inner radius of the coil in Fig. 10 for the no-flip case. Note that the momentum acceptance can be increased by increasing $a$, although the beta function under the coil also increases.

In the case when $p_{2}$ is kept fixed, the values of the beta function at the boundary and midplane are insensitive to changes in the coil thickness. As the current density and the resulting magnetic field is varied, the location of the second passbands changes, as we saw in the previous section, but the minimum value of the beta function in the center of the passband remains constant.

For the cases considered here, the minimum value of the beta function at the center of the passband is proportional to the width of the band. For a given coil length the no-flip configuration generates much wider passbands than the flip configuration. In general, it is difficult to find solutions that have both a low beta function and a wide momentum acceptance.

\section{Peak field enhancement}

The peak field decreases with increasing cell length for the no-flip case if the coil dimensions remain the same. The peak field for both cases increases with the length of the coil. Figure 11 shows the dependence of the peak field enhancement on the length of the coil. The peak field enhancement decreases as the coil is made longer if the other dimensions remain the same. The enhancement is larger when the polarity flips in alternate cells. The peak field for both cases decreases with increasing radius of the coil. The peak field for a given radius is smaller for the case where the polarity flips in alternate cells.

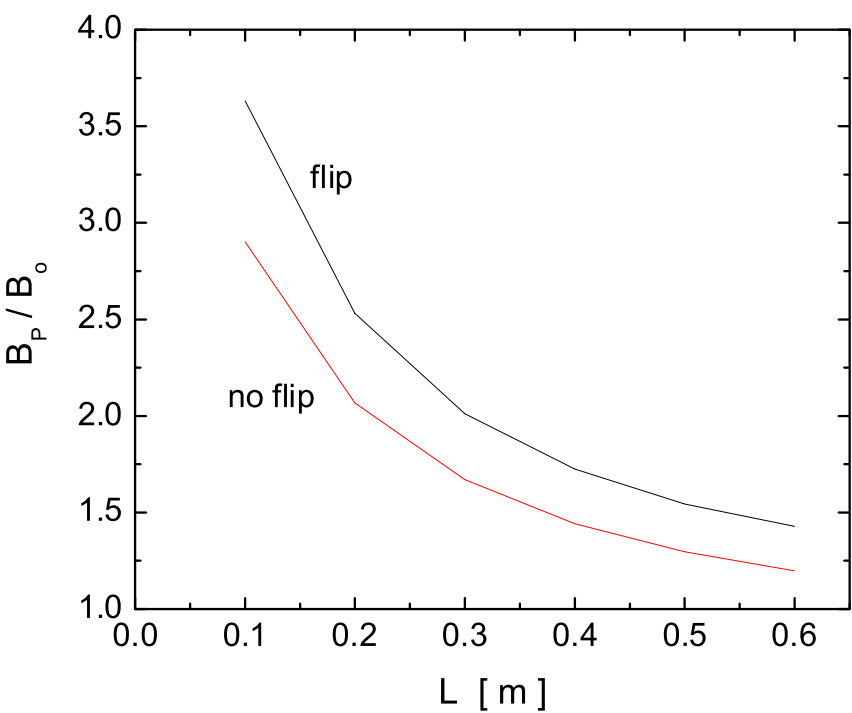

FIG. 11. (Color) Peak field enhancement as a function of the length of the coil. The other parameter values were $d=100 \mathrm{~cm}$, $a=40 \mathrm{~cm}, t=10 \mathrm{~cm}$, and $J=100 \mathrm{~A} / \mathrm{mm}^{2}$.

The central field and the peak field in the coil grow linearly with the current density. For a given current density, the central and peak fields are larger for the no-flip case. The central field and the peak field in the coil also grow approximately linearly for coil thicknesses for the parameter range considered here. For a given radial thickness the central and peak fields are larger for the no-flip case.

\section{E. Representative lattices}

We first look at solutions that use the high-energy passband. All momenta greater than $p_{\pi}$ are transmitted by the lattice. We see from Fig. 7 that the minimum beta function increases steadily as the momentum increases. Since we want a reference momentum $\left(p_{0}\right)$ around $200 \mathrm{MeV} / c$ and a momentum acceptance $\pm 100 \mathrm{MeV} / c$, our primary fitting criterion is to find solutions with $p_{\pi}=100 \mathrm{MeV} / c$. Solution A1 uses large-radius coils that could fit outside the rf cavities and that have constant polarity. This is an example of an $\langle+\mid+\rangle_{1}$ lattice. Both the minimum values of the beta function and the magnetic field occurs at the boundaries of the cell. The beta function is very flat with a minimum value of $65 \mathrm{~cm}$ and a maximum value of $67 \mathrm{~cm}$. Solution A2 also uses large-radius coils, but has alternating polarity. It is an example of an $\langle+\mid-\rangle_{1}$ lattice. Both the minimum values of the beta function and the magnetic field occur at the boundaries of the cell. The minimum value of the beta function is $72 \mathrm{~cm}$ and the maximum value is $87 \mathrm{~cm}$.

Solutions that make use of the second passband produce much smaller values of the beta function than those in the first passband. For the same application described earlier, our primary fitting criterion now is to find solutions with 
TABLE III. Summary of the one-cell lattice solutions

\begin{tabular}{lcccccc}
\hline \hline & sine & A1 & A2 & A3 & A4 & Study 2a \\
\hline Class & $\langle+\mid-\rangle_{1}$ & $\langle+\mid+\rangle_{1}$ & $\langle+\mid-\rangle_{1}$ & $\langle+\hat{+}+\rangle_{2}$ & $\langle-\hat{+}-\rangle_{2}$ & $\langle+\mid-\rangle_{1}$ \\
$d[\mathrm{~cm}]$ & 100 & 100 & 100 & 200 & 200 & 75 \\
$L[\mathrm{~cm}]$ & 67.1 & 30 & 40 & 74 & 80 & 15 \\
$a[\mathrm{~cm}]$ & 43.6 & 70 & 70 & 50 & 50 & 35 \\
$t[\mathrm{~cm}]$ & 12.6 & 6 & 14 & 25 & 35 & 15 \\
$J\left[\mathrm{~A} / \mathrm{mm}^{2}\right]$ & 40 & 90 & 91 & 27 & 26 & 107 \\
$p_{\pi}[\mathrm{MeV} / c]$ & 116 & 100 & 100 & & & 85 \\
$p_{2}[\mathrm{MeV} / c]$ & & & & 200 & 201 & \\
$\Delta p_{2}[\mathrm{MeV} / c]$ & & & & 87 & 64 & \\
$p_{0}[\mathrm{MeV} / c]$ & 200 & 200 & 200 & 200 & 200 & 220 \\
$\beta_{\text {abs }}\left(p_{0}\right)[\mathrm{cm}]$ & 58 & 65 & 72 & 16 & 9 & 73 \\
$L_{\mathrm{rf}}[\mathrm{cm}]$ & 32 & 100 & 100 & 126 & 120 & 60 \\
$B_{0}[\mathrm{~T}]$ & 2.8 & 2.1 & 2.4 & 4.7 & 5.5 & 2.8 \\
$B_{p}[\mathrm{~T}]$ & 4.1 & 4.3 & 7.9 & 6.0 & 7.1 & 7.5 \\
\hline \hline
\end{tabular}

the center of the band around $200 \mathrm{MeV} / c$. Ideally we would like the total width of the band to also be $\sim 200 \mathrm{MeV} / c$, so the accepted momentum range would be $100-300 \mathrm{MeV} / c$, but this has not been achieved in practice.

Solution A3 has a constant polarity lattice and is an example of a $\langle+\hat{+}+\rangle_{2}$ lattice. Since the field does not change polarity, we would eventually have to stop this lattice when the canonical angular momentum builds up. A polarity-reversing matching section would need to be provided and then the same type of lattice could be continued with the opposite sign of the field. The minimum value of the beta function, which is $16 \mathrm{~cm}$, occurs in the middle of the cell. The beta function rises to $80 \mathrm{~cm}$ at the cell boundaries. Solution A4 has an alternating-polarity lattice and is an example of a $\langle-\hat{+}-\rangle_{2}$ configuration. The minimum value of the beta function, which is $9 \mathrm{~cm}$, occurs in the middle of the cell. The beta function rises to $75 \mathrm{~cm}$ at the cell boundaries.

Typical parameters are shown in Table III. The quantity $\beta_{\text {abs }}$ is the beta function at the absorber location, which can be either at the boundary or the midplane depending on the solution. The quantity $L_{\mathrm{rf}}$ is an approximation of the space available for a $200 \mathrm{MHz}$ rf cavity. The last column shows the parameters for a single coil per cell design that was used for the cooling channel in U.S. Study 2a for a neutrino factory [25]. For this and other examples from the literature in the following sections, the fields and lattice parameters are the results of the calculations described here and sometimes differ slightly from the results presented in the original papers.

\section{LATTICES WITH TWO SOLENOIDS PER CELL}

We now consider cooling lattices with two solenoid coils in each geometric cell. In the most general case, the two coils could be totally different and this would introduce many more parameters than were available with one coil per cell. However, we will restrict our consideration to cases where the two coils have the same length and radii and have the same magnitudes of current density. In this case we allow an additional internal symmetry factor $f_{i}$ that specifies whether the two coils internal to the unit cell have the same $\left(f_{i}=1\right)$ or opposite $\left(f_{i}=-1\right)$ polarity. There is also one additional continuous parameter $g$ that specifies the gap separation of the coils from their nearest symmetric neighbor coil across the cell boundary, as shown in Fig. 2. Thus in this section we will examine the properties of lattices based on the eight parameters in the set $\left\{d, L, a, t, J, g ; f_{e}, f_{i}\right\}$. Solutions must satisfy the constraint $g+2 L<d$.

In order to uniquely specify the distance $g$, we start by picking any coil $\mathrm{C}$. Call the distance from $\mathrm{C}$ to the nearest coil on the left $d_{L}$ and the distance from $\mathrm{C}$ to the nearest coil on the right $d_{R}$. Then we define the gap $(g)$ as $g=$ $\min \left\{d_{L}, d_{R}\right\}$. The maximum value of the gap is then

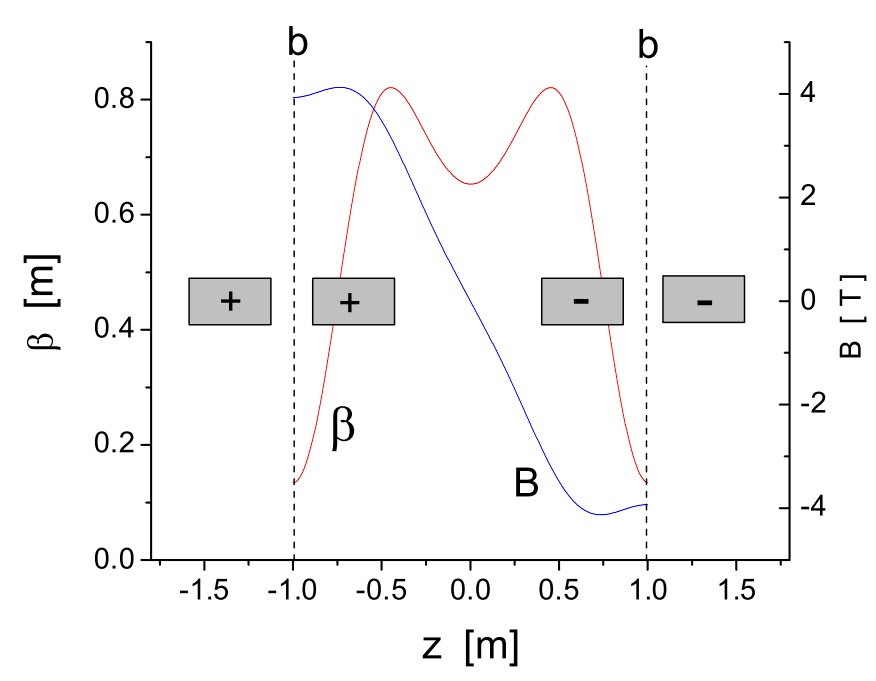

FIG. 12. (Color) An example of a $\langle+-\mid-+\rangle_{2}$ lattice. 
$g_{\max }=d / 2-L$. We define the cell boundary $(b)$ to be the axial location that bisects the gap, i.e. $b$ is at $g / 2$. In the case of equally spaced coils where $d_{L}=d_{R}$, there is an ambiguity in the definition of the lattice notation. For example, the sequence of coils ++--++ could be denoted either by $\langle++\mid--\rangle$ or by $\langle+-\mid-+\rangle$. However, both of these lattice classes produce the same result in this limit. This special case does not reduce to a lattice with one coil per geometric cell.

In order to make the meaning of this notation clearer, we show in Fig. 12 the coil locations, magnetic field, and beta function for one of the example lattices (B8) that we will discuss later. The boundary locations of the unit cell are marked with $b$. There is a + and a - polarity coil inside the unit cell. The coil polarity remains constant across the cell boundaries.

Having two coils per cell allows more flexibility in cooling lattice design. As we discuss below, it is often possible to change the momentum acceptance in the second passband by varying $g$. This also changes the location of the passband, but $J$ can then be adjusted to recenter the band.

\section{A. Thin lens matrix analysis}

Consider first the thin lens analysis of the two-coil cell. If we break the cell down to

$$
M=D\left(\frac{g}{2}\right) F(f) D(d-g) F(f) D\left(\frac{g}{2}\right),
$$

we find that the phase advance per cell is given by

$$
\cos \psi=1-\frac{d}{f}+\frac{g d}{2 f^{2}}-\frac{g^{2}}{2 f^{2}} .
$$

Note the quadratic dependence of the phase advance on the distance $g$. The minimum value of the beta function is

$$
\beta_{\min }=\frac{1}{\sin \psi}\left(d-\frac{g d}{f}+\frac{g^{2}}{2 f}+\frac{g^{2} d}{4 f^{2}}-\frac{g^{3}}{4 f^{2}}\right) .
$$

If we instead break the cell down as

$$
M=F\left(\frac{f}{2}\right) D(d-g) F(f) D(g) F\left(\frac{f}{2}\right),
$$

we find the maximum value of the beta function is

$$
\beta_{\max }=\frac{1}{\sin \psi}\left(d-\frac{g d}{f}+\frac{g^{2}}{f}\right) .
$$

\section{B. Lattice properties}

In general, a rich variety of behavior is seen in the twocoil configuration making it more difficult to specify simple rules for predicting the lattice properties. The location of the $\pi$ resonance increases for all symmetry classes for

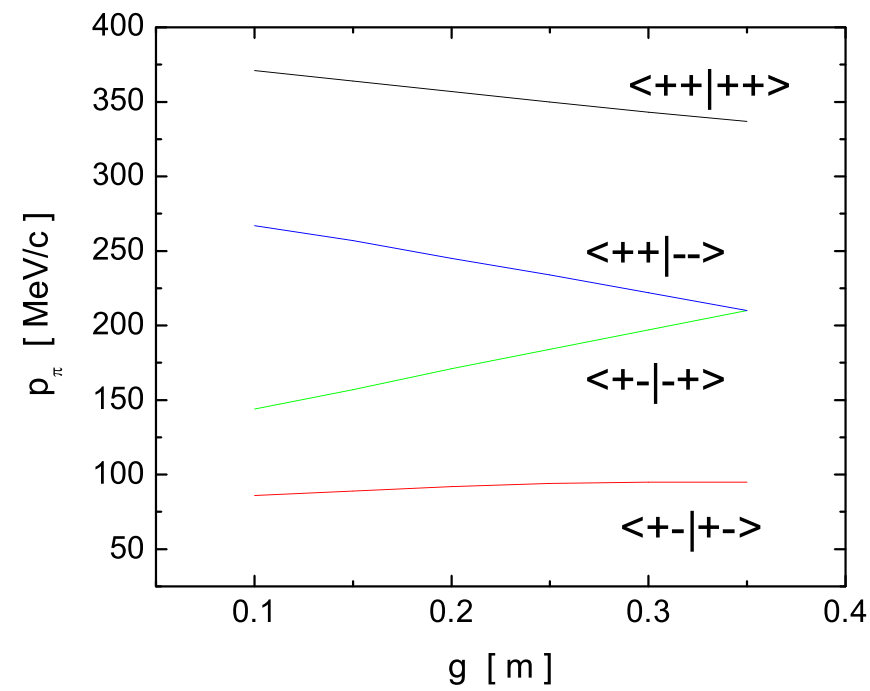

FIG. 13. (Color) Location of $\pi$ resonance as a function of $g$. The other parameter values were $d=150 \mathrm{~cm}, L=40 \mathrm{~cm}, a=$ $50 \mathrm{~cm}, t=10 \mathrm{~cm}$, and $J=70 \mathrm{~A} / \mathrm{mm}^{2}$.

increasing $L, t$, and $J$ when the other dimensions are held constant, while it decreases for increasing $a$. For changes in $d$ the location of the $\pi$ resonance increases when $f_{i}=-1$ and is approximately constant when $f_{i}=$ +1 . The dependence on $d$ is particularly strong for the $\langle+-\mid-+\rangle$ symmetry class.

The dependence of the $\pi$ resonance on the distance $g$ is shown in Fig. 13. Varying the gap between the coils across the boundary has little effect on the locations of the $\pi$ resonance for the symmetry classes with $f_{e}=+1$, but it

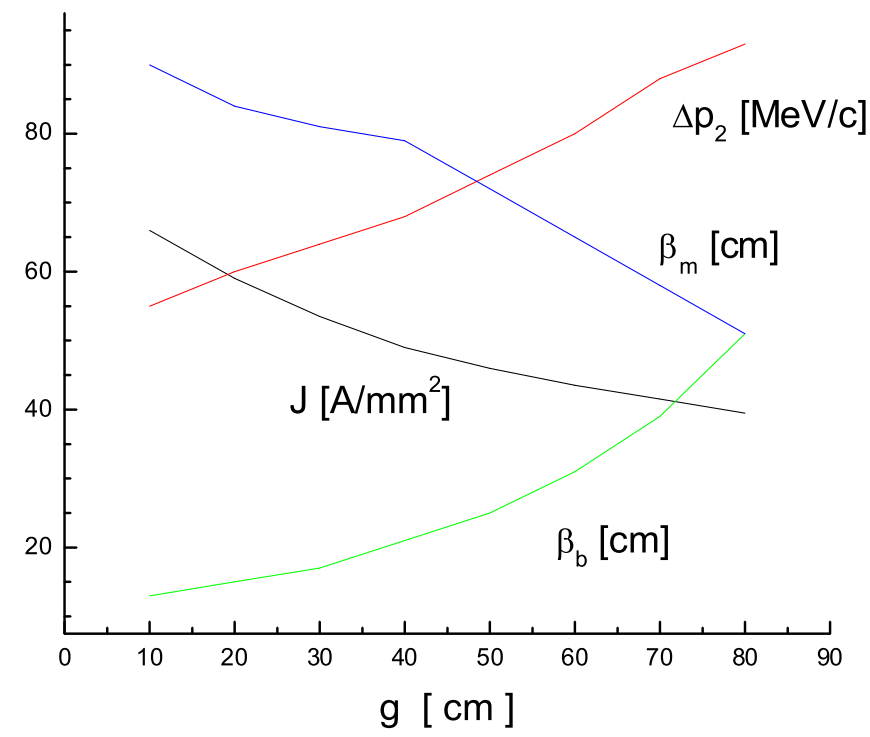

FIG. 14. (Color) Width of the second passband, and beta function at the cell boundary and cell midplane as a function of $g$ for the $\langle++\mid--\rangle$ symmetry class. The other parameter values were $d=275 \mathrm{~cm}, L=50 \mathrm{~cm}, a=35 \mathrm{~cm}$, and $t=11 \mathrm{~cm}$. 


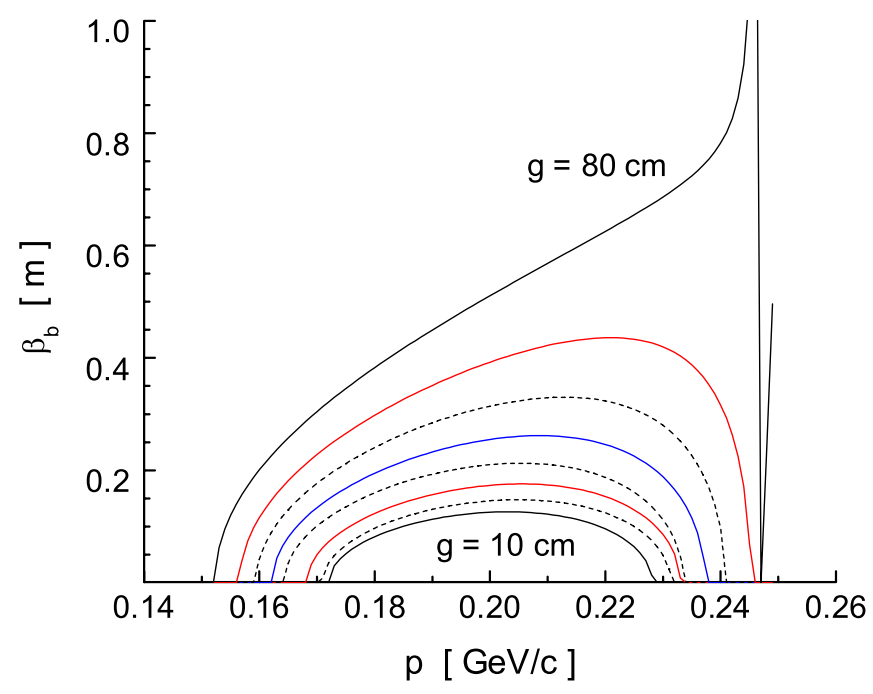

FIG. 15. (Color) Beta function at the cell boundary as a function of $p$ for the $\langle++\mid--\rangle$ symmetry class. The value of $g$ starts at $10 \mathrm{~cm}$, then increases in increments of $10 \mathrm{~cm}$. The other parameter values were $d=275 \mathrm{~cm}, L=50 \mathrm{~cm}, a=35 \mathrm{~cm}$, and $t=11 \mathrm{~cm}$.

has a very strong effect for the other two classes. Note the degenerate behavior of the $\langle++\mid--\rangle$ and $\langle+-\mid-+\rangle$ symmetry classes at $g_{\max }$.

We now look at the behavior of the properties of the second passband under the constraint that $p_{2}$ is fixed at $200 \mathrm{MeV} / c$. Figure 14 shows the width of the passband, and the beta functions at the cell boundary and at the cell midplane as a function of $g$ for the $\langle++\mid--\rangle$ symmetry class. We also show the value of the current density required to keep $p_{2}$ constant. The distance $g$ can be used to adjust the momentum acceptance of the band. As $g$ decreases the beta function at the boundary also decreases, while the beta function at the midplane increases. Note that $\beta_{b}$ is smaller than $\beta_{m}$ here, unlike the case for one coil per cell. The effects of varying $g$ on the momentum acceptance can be seen more clearly in Fig. 15. For small $g$ the acceptance is symmetric around $p_{2}$. For larger $g$ the acceptance increases. However, as the value of $g$ approaches $g_{\max }$ the focusing becomes too weak, the acceptance becomes distorted, and the beta function becomes very large at the upper end of the band. This distortion also occurs when $a$ is made large.

\section{Peak field}

The peak field in the coil increases for all symmetry classes for increasing $L, t$, and $J$ when the other dimensions are held constant. The peak field decreases for increasing $a$. For changes in $d$ the peak field increases when $f_{i}=-1$ and decreases when $f_{i}=+1$. The peak field for all cases goes up as $g$ is decreased, which corresponds to the case where the two neighbor coils across the cell boundary come close together.
TABLE IV. Summary of the two-cell high-energy passband characteristic solutions.

\begin{tabular}{lrrrrrr}
\hline \hline & B1 & B2 & \multicolumn{1}{c}{ B3 } & \multicolumn{1}{c}{ B4 } & \multicolumn{1}{c}{ B5 } & Study 1 \\
\hline Class & \multicolumn{1}{c}{$\mathrm{a}$} & \multicolumn{1}{c}{$\mathrm{c}$} & $\mathrm{c}$ & \multicolumn{1}{c}{$\mathrm{d}$} & \multicolumn{1}{c}{$\mathrm{e}$} & $\mathrm{c}$ \\
$d[\mathrm{~cm}]$ & 150 & 150 & 150 & 150 & 150 & 110 \\
$L[\mathrm{~cm}]$ & 20 & 25 & 20 & 20 & 20 & 30 \\
$a[\mathrm{~cm}]$ & 37 & 35 & 37 & 37 & 36 & 68 \\
$t[\mathrm{~cm}]$ & 5 & 10 & 10 & 10 & 6 & 35 \\
$J\left[\mathrm{~A} / \mathrm{mm}^{2}\right]$ & 66 & 77 & 81 & 92 & 79 & 49 \\
$g[\mathrm{~cm}]$ & 20 & 15 & 30 & 20 & 45 & 30 \\
$p_{\pi}[\mathrm{MeV} / c]$ & 97 & 100 & 101 & 100 & 100 & 156 \\
$p_{0}[\mathrm{MeV} / c]$ & 200 & 200 & 200 & 200 & 200 & 200 \\
$\beta_{\text {abs }}\left(p_{0}\right)[\mathrm{cm}]$ & 124 & 112 & 94 & 100 & 118 & 42 \\
$L_{\mathrm{rf}}[\mathrm{cm}]$ & 90 & 85 & 80 & 90 & 65 & 80 \\
$B_{0}[\mathrm{~T}]$ & 1.5 & 1.9 & 1.9 & 1.8 & 1.6 & 3.4 \\
$B_{p}[\mathrm{~T}]$ & 2.7 & 5.0 & 4.8 & 5.7 & 3.4 & 8.4 \\
\hline \hline
\end{tabular}

${ }^{\mathrm{a}}\left\langle++^{\wedge}++\right\rangle_{1} \cdot{ }^{\mathrm{b}}\left\langle-+^{\wedge}-+\right\rangle_{1} \cdot{ }^{\mathrm{c}}\langle++\mid--\rangle_{1}$.

$\mathrm{d}\left\langle-+^{\wedge}+-\right\rangle_{1} \cdot{ }^{\mathrm{e}}\left\langle++^{\wedge}--\right\rangle_{1}$.

\section{Representative lattices}

We have used the example initial cooling problem from the previous section to search for representative two-coil solutions. We try to find small radius solutions with the coils located between the rf cavities. For solutions with the minimum value of the beta function at the cell boundary we would locate the rf cavity at the cell midplane, whereas for solutions with the minimum of the beta function at the midpoint of the cell we would locate the rf cavity across the cell boundary between adjacent cells.

A summary of characteristic solutions for the highenergy passband is given in Table IV. All of the $B$ solutions in Table IV have the $\pi$ resonance located around $100 \mathrm{MeV} / c$, so the half-width of the momentum acceptance is also $100 \mathrm{MeV} / c$. Note that solutions for a given passband and given coil current density polarities can have

TABLE V. Summary of the two-cell characteristic solutions for the second momentum passband.

\begin{tabular}{lrrrcc}
\hline \hline & B6 & B7 & B8 & B9 & RFOFO \\
\hline Class & \multicolumn{1}{c}{ a } & \multicolumn{1}{c}{$\mathrm{b}$} & $\mathrm{c}$ & $\mathrm{d}$ & $\mathrm{b}$ \\
$d[\mathrm{~cm}]$ & 200 & 200 & 200 & 200 & 275 \\
$L[\mathrm{~cm}]$ & 34 & 47 & 47 & 46 & 50 \\
$a[\mathrm{~cm}]$ & 40 & 40 & 40 & 40 & 77 \\
$t[\mathrm{~cm}]$ & 16 & 20 & 16 & 20 & 11 \\
$J\left[\mathrm{~A} / \mathrm{mm}^{2}\right]$ & 42 & 47 & 40.5 & 36.8 & 95.3 \\
$g[\mathrm{~cm}]$ & 44 & 27 & 27 & 40 & 60 \\
$p_{2}[\mathrm{MeV} / c]$ & 200 & 199 & 201 & 200 & 198 \\
$\Delta p_{2}[\mathrm{MeV} / c]$ & 97 & 84 & 74 & 97 & 88 \\
$p_{0}[\mathrm{MeV} / c]$ & 200 & 200 & 200 & 200 & 200 \\
$\beta_{\mathrm{abs}}\left(p_{0}\right)[\mathrm{cm}]$ & 24 & 24 & 13 & 30 & 40 \\
$L_{\mathrm{rf}}[\mathrm{cm}]$ & 82 & 79 & 79 & 68 & 215 \\
$B_{0}[\mathrm{~T}]$ & 3.6 & 3.9 & 4.1 & 3.6 & 2.7 \\
$B_{p}[\mathrm{~T}]$ & 5.3 & 6.5 & 5.5 & 5.4 & 7.4 \\
\hline \hline
\end{tabular}

${ }^{\mathrm{a}}\langle++\mid++\rangle_{2} \cdot{ }^{\mathrm{b}}\langle+-\mid+-\rangle_{2} \cdot{ }^{\mathrm{c}}\langle+-\mid-+\rangle_{2} \cdot{ }^{\mathrm{d}}\left\langle-+^{\wedge}+-\right\rangle_{2}$. 
different beta function symmetries. Most of these solutions, other than B3, have the wrong natural symmetry for optimal cooling. Nevertheless in the table we assume the absorber is located at the cell boundary, which is the center of the intercell gap. The quantity $\beta_{\text {abs }}$ is the beta function at the absorber. In most cases this is not the minimum value of the beta function in the cell. Here the quantity $L_{\mathrm{rf}}$ is the larger intercoil spacing in the cell, which is centered on the cell midplane. The B3 solution appears best for cooling purposes. It has a beta function at the absorber location of $94 \mathrm{~cm}$ at the reference momentum. There is an $80 \mathrm{~cm}$ gap available for locating the rf cavity. The peak field in the coil is less than $5 \mathrm{~T}$. The last column shows the parameters for the baseline cooling lattice from the U.S. Feasibility Study I for a neutrino factory [26,27].

A summary of characteristic solutions for the second momentum passband is given in Table V. All of the $B$ solutions in Table $\mathrm{V}$ have the center of the second passband located around $200 \mathrm{MeV} / c$. Three of these $B$ solutions have the beta minimum at the cell boundary, which is the correct natural symmetry for optimal cooling. These solutions have smaller beta functions at the absorber and less momentum acceptance than the solutions in Table IV. Solution B6 would be suitable for cooling, provided that occasional field-flip matching sections are added. The B8 solution appears best for cooling purposes. It has a beta function at the absorber location of $13 \mathrm{~cm}$ at the reference momentum, although the full momentum acceptance is only $74 \mathrm{MeV} / c$. There is a $79 \mathrm{~cm}$ space available for locating the rf cavity. The peak field in the coil is $5.5 \mathrm{~T}$. The B9 example shows that two-coil solutions also exist for the second passband with the minimum of the beta function at the cell midplane. The last column shows the parameters for the transverse cooling lattice that was used as the basis for the RFOFO cooling ring [28].

\section{LATTICES WITH THREE SOLENOIDS PER CELL}

We next consider cooling lattices with three solenoid coils in each geometric cell. In the most general case, the three coils could be totally different and this would introduce many more parameters than were available with two coils per cell. However, a simpler way to make use of the new coil is to use the current in the third coil as a means of adjusting the lattice properties without modifying the geometry. Thus, we will restrict our consideration to symmetric cases where the two outer coils have the same length, radii, and current density. We will denote these as the focusing coils. We assume the third coil, which we call the coupling coil, is located symmetrically between the focusing coils. We will place no restrictions on the length, radii, or current density of the coupling coil. The relative polarity of the current density between the focusing and coupling coils will be specified using an internal symmetry factor $f_{i}$. As before we use $f_{e}$ to indicate whether the overall polarity flips $\left(f_{e}=-1\right)$ or not $\left(f_{e}=1\right)$ in alternate cells. The layout of the geometric cell is shown in Fig. 2. There is one additional axial parameter $h$ that specifies the length of the coupling coil. Thus, in this section we will examine the properties of lattices based on the 12 parameters in the set $\left\{d, L, a, t, J, g, h, a_{c}, t_{c}, J_{c} ; f_{e}, f_{i}\right\}$. Solutions must satisfy the constraint $2 L+g+h<d$. We define the cell boundary $(b)$ to be the axial location that bisects the gap, i.e. $b$ is at $g / 2$. We define the cell midplane $(m)$ to be the axial position midway between cell boundaries, i.e., $m$ is at the center of the coupling coil. There is no ambiguity in the definition of the cell boundary in the case of equally spaced coils with the same dimensions and the same magnitude of the current density since we are requiring both focusing coils to have the same polarity.

Having two adjustable currents in each cell gives us a "tunable" lattice for the second passband. One can use the coupling coil $J_{c}$ to set the momentum acceptance and beta function at the absorber. Then the band can be recentered at the desired momentum by adjusting the focusing $J$. In this way the properties of the lattice can be adjusted without changing the size or location of the coils. For example, the beta function can be tapered to follow the reduction in beam emittance from cooling.

The thin lens analysis for this case contains many additional terms containing the focal length of the coupling coil and is not particularly enlightening. The peak field in these configurations can occur in either the focusing or the coupling coil. In terms of the new variables the peak field is not very sensitive to changes in $g$ or $a_{c}$. The peak field

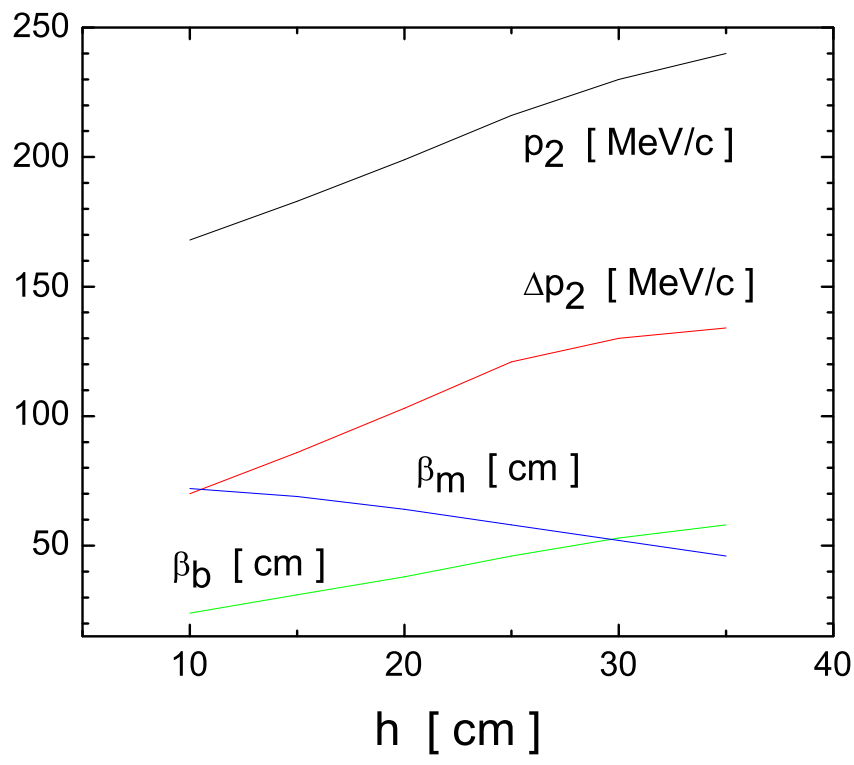

FIG. 16. (Color) Location of the center of the second passband $p_{2}$, the width of the band, the beta function at the cell boundary $\beta_{b}$, and the beta function at the cell midplane $\beta_{m}$ as a function of the distance $h$. The other parameter values were $d=2.5 \mathrm{~m}, L=$ $18 \mathrm{~cm}, a=35 \mathrm{~cm}, t=17 \mathrm{~cm}, J=73 \mathrm{~A} / \mathrm{mm}^{2}, g=54 \mathrm{~cm}$, $a_{c}=70 \mathrm{~cm}, t_{c}=12 \mathrm{~cm}$, and $J_{c}=75 \mathrm{~A} / \mathrm{mm}^{2}$. 


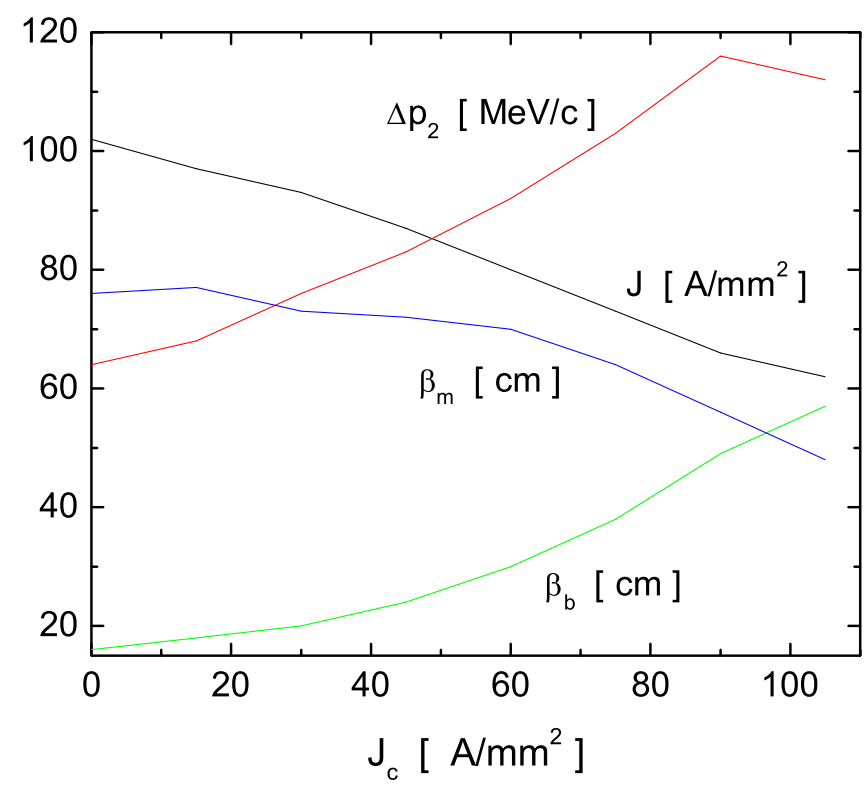

FIG. 17. (Color) The current density $J$ in the focusing coils, the width of the second passband, the beta function at the cell boundary $\beta_{b}$, and the beta function at the cell midplane $\beta_{m}$ as a function of the current density $J_{c}$ in the coupling coil. The other parameter values were $d=2.5 \mathrm{~m}, L=18 \mathrm{~cm}, a=$ $35 \mathrm{~cm}, t=17 \mathrm{~cm}, g=54 \mathrm{~cm}, h=20 \mathrm{~cm}, a_{c}=70 \mathrm{~cm}$, and $t_{c}=12 \mathrm{~cm}$.

does however grow approximately linearly with $h$ for all the symmetry classes. In general, the peak fields for $200 \mathrm{MeV} / c$ reference momentum solutions are smaller with three coils per cell than the corresponding solutions with one coil per cell.

\section{A. Lattice properties}

With the large number of available parameters, it is difficult to make completely general statements about the properties of the three-coil lattice configurations. The location of the $\pi$ resonance can be changed by varying the gap distance $g$ or the coupling coil length $h$. The $\pi$ resonance increases in momentum for increasing $h$ for all symmetry classes when the coupling coil is long.

The dependence of some properties of the second passband on the coupling coil length for the $\langle+++\mid---\rangle$ symmetry class are shown in Fig. 16. The location and width of the band increase with increasing $h$. The minimum value of the beta function occurs at the cell boundary for $h$ up to $\sim 30 \mathrm{~cm}$ for this example and then switches to the cell midplane for longer coils. This shows that the character of the solution can be changed by varying the length of the coupling coil. In this example we can only obtain a low value of $\beta_{b}$ by also accepting a low momentum acceptance. The dependence of some properties of the second passband on the current density in the coupling coil for the $\langle+++\mid---\rangle$ symmetry class is shown in Fig. 17. For this figure the current density in the focusing
TABLE VI. Summary of the three-cell characteristic solutions for the first passband.

\begin{tabular}{lrrrr}
\hline \hline & $\mathrm{C} 1$ & $\mathrm{C} 2$ & $\mathrm{C} 3$ & $\mathrm{C} 4$ \\
\hline Class & \multicolumn{1}{c}{$\mathrm{a}$} & $\mathrm{b}$ & $\mathrm{c}$ & $\mathrm{d}$ \\
$d[\mathrm{~cm}]$ & 200 & 200 & 200 & 200 \\
$L[\mathrm{~cm}]$ & 10 & 25 & 16 & 30 \\
$a[\mathrm{~cm}]$ & 40 & 75 & 39 & 76 \\
$t[\mathrm{~cm}]$ & 5 & 5 & 6 & 8 \\
$J\left[\mathrm{~A} / \mathrm{mm}^{2}\right]$ & 69 & 50 & 70 & 46 \\
$g[\mathrm{~cm}]$ & 50 & 50 & 38 & 30 \\
$h[\mathrm{~cm}]$ & 30 & 67 & 30 & 65 \\
$a_{c}[\mathrm{~cm}]$ & 70 & 35 & 70 & 35 \\
$t_{c}[\mathrm{~cm}]$ & 5 & 5 & 6 & 5 \\
$J_{c}\left[\mathrm{~A} / \mathrm{mm}^{2}\right]$ & 63 & 50 & 65 & 49 \\
$p_{\pi}[\mathrm{MeV} / c]$ & 99 & 100 & 101 & 101 \\
$p_{0}[\mathrm{MeV} / c]$ & 200 & 200 & 200 & 200 \\
$\beta_{\text {abs }}\left(p_{0}\right)[\mathrm{cm}]$ & 129 & 152 & 130 & 154 \\
$L_{\mathrm{rf}}[\mathrm{cm}]$ & 130 & 133 & 130 & 135 \\
$B_{0}[\mathrm{~T}]$ & 1.1 & 1.5 & 1.1 & 1.5 \\
$B_{p}[\mathrm{~T}]$ & 2.4 & 2.0 & 2.9 & 2.6 \\
\hline \hline
\end{tabular}

${ }^{\mathrm{a}}\langle+++\mid+++\rangle_{1} \cdot{ }^{\mathrm{b}}\langle+-+\mid+-+\rangle_{1} \cdot{ }^{\mathrm{c}}\langle+++\mid---\rangle_{1}$.

$\mathrm{d}\langle+-+\mid-+-\rangle_{1}$.

coils was adjusted to keep $p_{2}$ fixed at $200 \mathrm{MeV} / c$. The width of the band tends to grow for increasing coupling current. The minimum value of the beta function occurs at the cell boundary for $h$ up to $\sim 95 \mathrm{~A} / \mathrm{mm}^{2}$ for this example and then switches to the cell midplane for higher current densities. This shows that the character of the solution can also be changed by varying the relative currents in the coupling and focus coils.

TABLE VII. Summary of the three-cell characteristic solutions for the second passband.

\begin{tabular}{lrrrrrc}
\hline \hline & C5 & \multicolumn{1}{c}{ C6 } & \multicolumn{1}{c}{ C7 } & \multicolumn{1}{c}{ C8 } & \multicolumn{1}{c}{ C9 } & Study 2 \\
\hline Class & \multicolumn{1}{c}{$\mathrm{a}$} & \multicolumn{1}{c}{$\mathrm{b}$} & \multicolumn{1}{c}{$\mathrm{c}$} & \multicolumn{1}{c}{$\mathrm{d}$} & \multicolumn{1}{c}{$\mathrm{e}$} & $\mathrm{d}$ \\
$d[\mathrm{~cm}]$ & 250 & 250 & 250 & 250 & 250 & 275 \\
$L[\mathrm{~cm}]$ & 30 & 15 & 30 & 18 & 30 & 16.7 \\
$a[\mathrm{~cm}]$ & 75 & 35 & 75 & 35 & 35 & 33 \\
$t[\mathrm{~cm}]$ & 10 & 25 & 10 & 17 & 14 & 17.5 \\
$J\left[\mathrm{~A} / \mathrm{mm}^{2}\right]$ & 49 & 72 & 49 & 73 & 70 & 75.2 \\
$g[\mathrm{~cm}]$ & 30 & 88 & 30 & 54 & 70 & 35 \\
$h[\mathrm{~cm}]$ & 80 & 42 & 80 & 20 & 20 & 33 \\
$a_{c}[\mathrm{~cm}]$ & 35 & 74 & 35 & 70 & 70 & 77 \\
$t_{c}[\mathrm{~cm}]$ & 10 & 5 & 14 & 12 & 10 & 8 \\
$J_{c}\left[\mathrm{~A} / \mathrm{mm}^{2}\right]$ & 56 & -70 & 55 & 75 & 65 & 98 \\
$p_{2}[\mathrm{MeV} / c]$ & 201 & 199 & 199 & 199 & 201 & 194 \\
$\Delta p_{2}[\mathrm{MeV} / c]$ & 52 & 69 & 36 & 103 & 70 & 117 \\
$p_{0}[\mathrm{MeV} / c]$ & 200 & 200 & 200 & 200 & 200 & 200 \\
$\beta_{\text {abs }}\left(p_{0}\right)[\mathrm{cm}]$ & 7 & 19 & 4 & 38 & 22 & 49 \\
$L_{\mathrm{rf}}[\mathrm{cm}]$ & 170 & 132 & 170 & 160 & 120 & 205 \\
$B_{0}[\mathrm{~T}]$ & 5.3 & 3.5 & 6.2 & 3.1 & 3.6 & 2.8 \\
$B_{p}[\mathrm{~T}]$ & 6.0 & 7.1 & 7.2 & 6.4 & 6.5 & 6.4 \\
\hline \hline
\end{tabular}

${ }^{\mathrm{a}}\langle--+\hat{+}+--\rangle_{2} \cdot{ }^{\mathrm{b}}\langle+-+\mid+-+\rangle_{2} \cdot{ }^{\mathrm{c}}\langle-+-\hat{+}-+-\rangle_{2}$. $\mathrm{d}\langle+++\mid--\rangle_{2} \cdot \dot{\mathrm{e}}\langle+-+\mid-+-\rangle_{2}$. 


\section{B. Representative lattices}

We have used the example initial cooling problem from the previous sections to search for representative three-coil solutions. We try to find solutions where either the focusing coils or the coupling coil have small radius and are located between rf cavities. The other coil then has to have a large radius and be located outside the rf cavity. The most desirable solution with small focusing coil radius has the minimum of the beta function at the cell boundary, while solutions with small coupling coil radius want the minimum to occur at the cell midplane.

A summary of characteristic solutions for the highenergy passband is given in Table VI. The maximum length available for the rf cavity is $d-g-2 L$ for solutions with small focusing coil radius and $d-h$ for solutions with small coupling coil radius. None of these solutions offer any obvious advantage over lattices with one or two coils per cell.

A summary of characteristic solutions for the second passband is given in Table VII. Solution C8 is an attractive solution for early cooling. It is an $\langle+++\mid---\rangle_{2}$ configuration with a minimum beta function of $38 \mathrm{~cm}$ and a band width of $103 \mathrm{MeV} / c$. The last column shows the parameters for the second U.S. feasibility study for a neutrino factory $[3,29,30]$.

\section{SCALING RELATIONS}

Any of the lattice solutions discussed in the previous sections can be scaled geometrically to produce additional lattices that may be more satisfactory for other conditions. Suppose we scale all the cell dimensions by the same factor $f$. Then it follows from Eq. (4) that we can obtain the same on-axis field by scaling the current density by $1 / f$. However, we have seen in Eq. (8) that the passband loca-

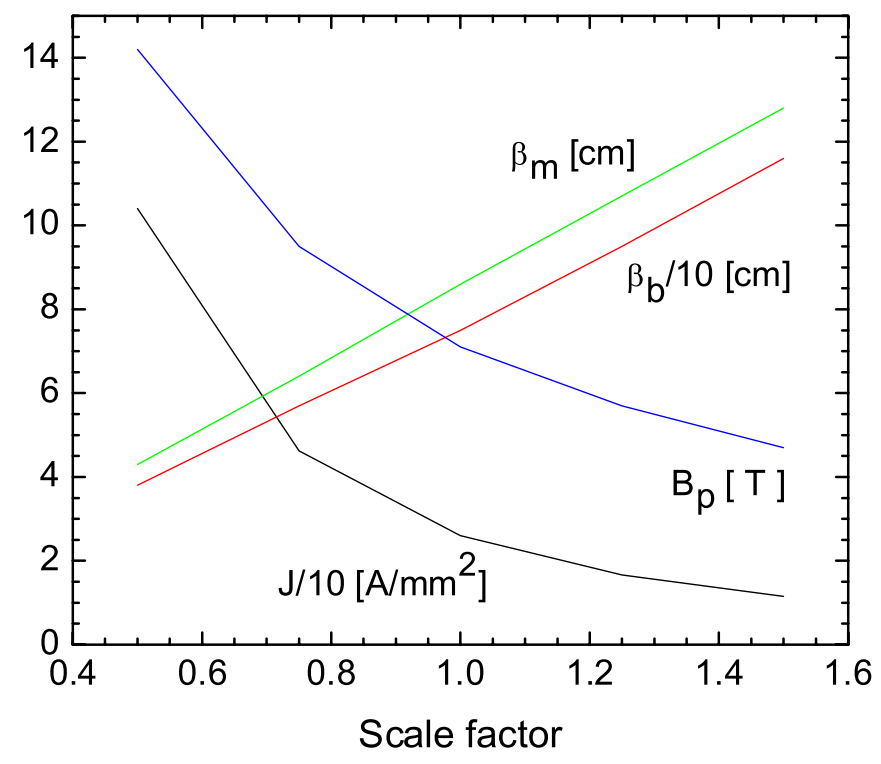

FIG. 18. (Color) Geometrical scaling of the A4 example lattice. tions scale like $B_{o} d$. Thus we need to scale the current density like $1 / f^{2}$ to keep the passband locations fixed. This is illustrated in Fig. 18 where the scale factor of 1 corresponds to the A4 example. The current density was adjusted to keep $p_{2}$ fixed at $200 \mathrm{MeV} / c$. The peak field in the coil falls with increasing scale factor. The maximum onaxis field is proportional to the peak field in the coil. Both the beta function at the boundary and at the midplane grow linearly with increasing scale factor. However, note that the magnitude of the beta function at the midplane is still quite small ( $\sim 13 \mathrm{~cm}$ for a $3 \mathrm{~m}$ cell length). The momentum acceptance (not shown) is independent of the scale factor.

In the previous sections we have systematically examined the characteristics of a large number of cooling lattices. In order to compare solutions with different numbers of coils per cell and different symmetry classes, it is useful to introduce another scaling variable. We have seen that there is often a direct relationship between the magnitude of the beta function and the momentum acceptance. Since the beta function depends on the scaling parameter $f$, we can define a normalized beta function corresponding to a fixed maximum axial field. We then examine how this scaled beta function depends on the momentum acceptance.

We define the scaled momentum acceptance as

$$
\begin{aligned}
& \delta= \pm \frac{p-p_{\pi}}{p} \text { for the first passband } \\
& \delta= \pm \frac{\Delta p_{2}}{2 p_{2}} \text { for the second passband. }
\end{aligned}
$$

We define the scaled beta function $F_{1}$ as the actual beta function for some lattice normalized to the value of the

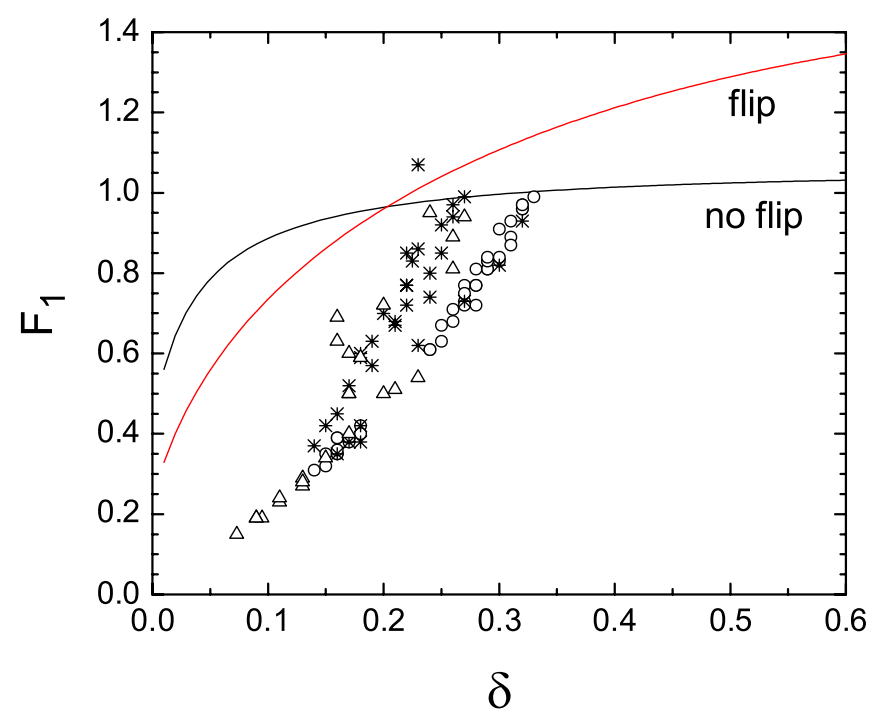

FIG. 19. (Color) Scaled beta function as a function of scaled acceptance. The solid lines show first passband results for the flip (A2) and no-flip (A1) example lattices. Symbols show second passband solutions with $p_{2}=200 \mathrm{MeV} / c ; \bigcirc: 1$ coil per cell, *: 2 coils per cell, $\triangle: 3$ coils per cell. 


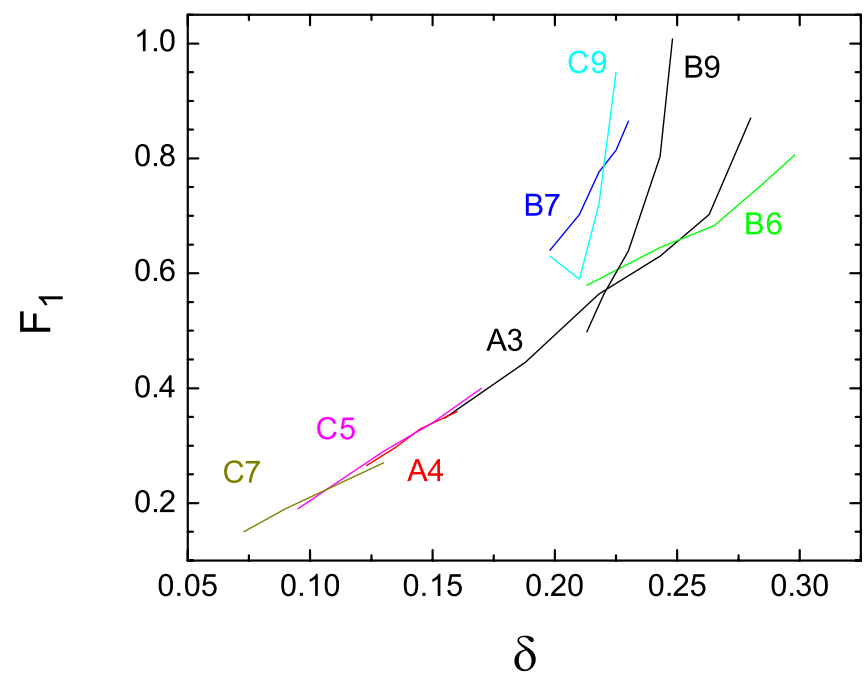

FIG. 20. (Color) Scaled beta function as a function of scaled acceptance for example lattices for the second passband centered at $200 \mathrm{MeV} / c$.

beta function for the continuous solenoid evaluated with the same value of the maximum on-axis field. Using Eq. (3)

$$
F_{1}=\frac{\beta}{\beta_{o}}=\beta \frac{e B_{o}}{2 p}
$$

The scaled behavior of some of the example lattices discussed previously are shown in Fig. 19. Large regions of the first passband solutions and most of the second passband solutions have $F_{1}$ smaller than 1 and thus produce more efficient cooling than a continuous solenoid. In the first passband alternating-polarity lattices are more advantageous for small momentum acceptance. At the beginning of cooling the momentum spread of the beam is large. In this case, one would use a lattice operating in the first passband because any value of the momentum acceptance is possible in principle by operating far enough away from $p_{\pi}$. However, the beta functions for these lattices are also large. After a sufficient amount of longitudinal cooling reduces the momentum spread to $\sim \pm 30 \%$ full width, one can switch to a lattice that uses the second passband and has smaller beta functions at the absorbers. The scaled behavior of some of the example lattices discussed previously are shown in Fig. 20. For each of these example solutions the radius was changed to vary the momentum acceptance and the current density was adjusted to recenter the band at $200 \mathrm{MeV} / c$. The strong correlation between the minimum beta function and the momentum acceptance can be clearly seen in this figure.

One can proceed through a number of these stages, leading finally to a lattice with small acceptance and very small beta function. For $\delta \geq 10 \%$, using current $25 \mathrm{~T}$ high temperature superconductor technology, the smallest beta function using solenoid focusing appears to be $\sim 1 \mathrm{~cm}$.

\section{SUMMARY}

Ionization cooling channels play an important role in the design of neutrino factories and muon colliders. In this paper we have summarized the status of cooling lattice design using periodic solenoid focusing. There is considerable flexibility in the design of these channels and they exhibit a great variety of interesting properties. The detailed behavior of the lattice properties is strongly influenced by the symmetries exhibited by the polarities of the currents in the coils in the periodic channel. In order to describe this behavior, we introduced a new system for classifying the symmetry properties of periodic solenoid cooling lattices. A method using symplectic integration of the one-cell transport matrix was used to calculate momentum stop bands and beta functions. This integration used the on-axis field calculated from solenoid coil blocks. We found this method was accurate by showing that a sinusoidally varying magnetic field gave results in good agreement with predictions from solutions of the Mathieu equation. The peak field in the coil was an important design constraint. We showed how the peak field in the coil and the lattice properties depend on the geometric properties of the coils.

We introduced a new optimization procedure that emphasizes obtaining a desired momentum band and minimizing the peak field in the coil. We used this method to systematically search for lattice configurations that could be used in a neutrino factory or muon collider. We saw that most features of the lattice dynamics could be seen even in the simplest case of a single coil per geometric cell. There are two momentum passbands that have been used for cooling lattices. The beta function in the high momentum band is determined mainly by the amount of momentum acceptance that is required. The location of the $\pi$ resonance scales with the maximum value of the on-axis magnetic field. The minimum value of the beta function and the momentum acceptance are typically much smaller in the second passband. The momentum acceptance can be adjusted by varying the radius and length of the coil.

Adding a second coil per geometric cell adds considerable flexibility for cooling channel design. The gap between the two coils across the cell boundary is an important design parameter that can have strong effects on the momentum acceptance and other lattice properties. In addition, the number of possible symmetry classes is doubled. With three coils per cell it is possible to design a tunable lattice with fixed coil positions and radii. The focusing coil current is used to set the acceptance and the beta function value at the absorber, while the coupling coil current is used to center the location of the passband. The properties of different lattice types were compared using a scaled value of the beta function. The value of the beta function is directly related to the momentum acceptance. For all the coil configurations we have produced sample solutions for each of the symmetry classes. Several of these 
lattice designs have interesting properties and might be suitable for further investigation for the transverse cooling channel of a neutrino factory or muon collider.

\section{ACKNOWLEDGMENTS}

We would like to thank G. Penn, J.S. Berg, and V. Balbekov for useful discussions on some of the topics covered in this paper. This effort was supported by the Office of High Energy Physics of the U.S. Department of Energy under Contract No. DE-AC02-98CH10886.

[1] A. N. Skrinsky and V. V. Parkhomchuk, Sov. J. Part. Nucl. 12, 223 (1981).

[2] D. Neuffer, Part. Accel. 14, 75 (1983).

[3] M. M. Alsharo'a et al., Phys. Rev. ST Accel. Beams 6, 081001 (2003).

[4] C. M. Ankenbrandt et al., Phys. Rev. ST Accel. Beams 2, 081001 (1999).

[5] R. Palmer et al., Nucl. Phys. B, Proc. Suppl. 51A, 61 (1996).

[6] E.-S. Kim, C. Kim, G. Penn, A. Sessler, and J. Wurtele (Neutrino Factory and Muon Collider Collaboration), Technical Report 36 (1999), http://nfmcc-docdb.fnal.gov/ cgi-bin/DocumentDatabase.

[7] S. Lee, Accelerator Physics (World Scientific, Singapore, 1999).

[8] S. Gilardoni (CERN Neutrino Factory Collaboration), Technical Report 107 (2002), http://slap.web.cern.ch/ slap/NuFact/NuFact/NFNotes.html.

[9] G. Penn and J. Wurtele, Phys. Rev. Lett. 85, 764 (2000).

[10] G. Penn (Neutrino Factory and Muon Collider Collaboration), Technical Report 71 (2000), http:// nfmcc-docdb.fnal.gov/cgi-bin/DocumentDatabase.

[11] R. Fernow (Neutrino Factory and Muon Collider Collaboration), Technical Report 244 (2002), http:// nfmcc-docdb.fnal.gov/cgi-bin/DocumentDatabase.

[12] C. Wang and K. Kim, Phys. Rev. E 63, 056502 (2001).

[13] R. Palmer and R. Fernow, U.S. Particle Accelerator School, Vanderbilt University, http://pubweb.bnl.gov/ users/palmer/www/course.
[14] R. B. Palmer, Technical Report 22, Brookhaven National Laboratory, 1998, http://pubweb.bnl.gov/users/palmer/ www/notes/whyalt.ps.

[15] M. Reiser, Theory and Design of Charged Particle Beams (Wiley, New York, 1994).

[16] M. W. Garrett, J. Appl. Phys. 34, 2567 (1963).

[17] A. M. Clogston and H. Heffner, J. Appl. Phys. 25, 436 (1954).

[18] G. Caporaso and A. Cole, in The Physics of Particle Accelerators, AIP Conf. Proc. No. 249 (AIP, New York, 1992), pp. 1615-1712.

[19] R. Fernow (Neutrino Factory and Muon Collider Collaboration), Technical Report 23 (1999), http:// nfmcc-docdb.fnal.gov/cgi-bin/DocumentDatabase.

[20] B. L. Militsyn, C. van der Geer, and W. Urbanus, in Proceedings of the 2000 European Particle Accelerator Conference (2000), pp. 1054-1056.

[21] M. Preto and S. Tremaine, Astron. J. 118, 2532 (1999).

[22] A. Chao and M. Tigner, Handbook of Accelerator Physics and Engineering (World Scientific, Singapore, 1999), p. 402.

[23] M. Green (Neutrino Factory and Muon Collider Collaboration), Technical Report 165 (2000), http:// nfmcc-docdb.fnal.gov/cgi-bin/DocumentDatabase.

[24] V. Balbekov (Neutrino Factory and Muon Collider Collaboration), Technical Report 190 (2001), http:// nfmcc-docdb.fnal.gov/cgi-bin/DocumentDatabase.

[25] J. Berg et al., Phys. Rev. ST Accel. Beams 9, 011001 (2006).

[26] Technical Report Fermilab-Pub-00/108-E, Fermilab, 2000, edited by N. Holtkamp and D. Finley, http://www.fnal.gov/projects/muon_collider/nu-factory/ nu-factory.html.

[27] J. Monroe, P. Spentzouris, V. Balbekov, P. Lebrun, G. Penn, C. Kim, E. Kim, and D. Kaplan, Phys. Rev. ST Accel. Beams 4, 041301 (2001).

[28] R. Palmer et al., Phys. Rev. ST Accel. Beams 8, 061003 (2005).

[29] Technical Report, BNL-52623 (2001), edited by S. Ozaki, R. Palmer, M. Zisman, and J. Gallardo, http:// www.cap.bnl.gov/mumu/studyii/FS2-report.html.

[30] R. Fernow (Neutrino Factory and Muon Collider Collaboration), Technical Report 241 (2002), http:// nfmcc-docdb.fnal.gov/cgi-bin/DocumentDatabase. 